\title{
REPRESENTATION THEOREMS FOR NONLINEAR DISJOINTLY ADDITIVE FUNCTIONALS AND OPERATORS ON SOBOLEV SPACES(1)
}

\author{
BY
}

\author{
MOSHE MARCUS AND VICTOR J. MIZEL
}

\begin{abstract}
An abstract characterization is obtained for a class of nonlinear differential operators defined on the subspace $S=W_{k}^{p}[a, b]$ of the $k$ th order Sobolev space $W_{k}^{p}[a, b], 1 \leqslant k, 1 \leqslant p \leqslant \infty$. It is shown that every mapping $T: S \rightarrow L^{1}[a, b]$ which is local, continuous and $D^{k}$-disjointly additive has the form $(T u)(t)=H\left(t, D^{k} u(t)\right)$, where $H:[a, b] \times R \rightarrow R$ is a function obeying Carathéodory conditions as well as $(*) H(\cdot, 0)=0$. Here $D^{k}$-disjoint additivity means $T(u+v)=T u+T v$ whenever $\left(D^{k} u\right)\left(D^{k} v\right)=0$. Likewise, every real functional $N$ on $S$ which is continuous and $D^{k}$-disjointly additive has the form $N(u)=\int T u$, with $T$ as above. Liapunov's theorem on vector measures plays a crucial role, and the analysis suggests new questions about such measures. Likewise, a new type of Radon-Nikodým theorem is employed in an essential way.
\end{abstract}

0. Introduction. In this paper we develop a representation theory for certain classes of nonlinear functionals and nonlinear operators on the spaces $\dot{W}_{k}^{p}(I)$, where $k$ is a positive integer, $p$ satisfies $1 \leqslant p \leqslant \infty$, and $I$ is the interval $[0,1]$.

Recall that $W_{k}^{p}(I)$ denotes the Sobolev space

$$
W_{k}^{p}(I)=\left\{u \in L^{p}(I): D^{j} u \in L^{p}(I), 1 \leqslant j \leqslant k\right\},
$$

where $D^{j} u$ denotes the $j$ th order distribution derivative of $u$. (All functions here are taken to be real.) It is known that a function $u$ belongs to $W_{k}^{p}(I)$ if and only if it is equivalent to a function in $C^{k-1}(I)$ whose $(k-1)$ st derivative is absolutely continuous and whose $k$ th derivative belongs to $L^{p}(I)$. Hereafter any function from $W_{k}^{p}(I)$ will be assumed to be the continuous representative of its equivalence class. The space $W_{k}^{p}(I)$ is a Banach space under the norm

$$
\|u\|_{W_{k}^{p}(I)}=\sum_{j=0}^{k}\left\|D^{j} u\right\|_{L^{p}(I)} .
$$

Received by the editors August 25, 1975.

AMS (MOS) subject classifications (1970). Primary 46E35, 46G99; Secondary 34A99, 28 A45.

$K e y$ words and phrases. $D^{k}$-disjointly additive functional, local $D^{k}$-disjointly additive operator, normalized Carathéodory function, Liapunov vector measure, Radon-Nikodým type theorem.

(') This research was partially supported by the U.S National Science Foundation under Grant GP 28377A2. 
The subspace $\stackrel{\circ}{k}_{k}^{p}(I) \subset W_{k}^{p}$ is defined by

$$
\stackrel{\mathscr{W}}{k}^{p}(I)=\left\{u \in W_{k}^{p}(I): D^{j} u(0)=D^{j} u(1)=0,1 \leqslant j \leqslant k-1\right\} .
$$

In $\dot{W}_{k}^{p}(I)$ the norm $(0.1)$ is equivalent to

$$
|u|_{k, p}^{I}=\left\|D^{k} u\right\|_{L^{p}(I)} .
$$

It is well known that every continuous linear functional $L$ on $\dot{W}_{k}^{p}(I), 1 \leqslant p$ $<\infty$, has a representation of the form

$$
L u=\int_{I} g D^{k} u d m,
$$

where $g$ is a function in $L^{p^{\prime}}(I)\left(1 / p+1 / p^{\prime}=1\right)$ and $m$ denotes Lebesgue measure. This representation is also valid in the case $p=\infty$ if $L$ is $(b m)_{k}$ continuous, where $(\mathrm{bm})_{k}$ denotes the topology of bounded $k$-convergence on $W_{k}^{\infty}(I)$ which is defined as follows. A sequence $\left\{u_{n}\right\}$ is $(b m)_{k}$-convergent provided that it is bounded in $W_{k}^{\infty}$ norm and that $\left\{D^{j} u_{n}\right\}$ converges in measure, for $0 \leqslant j \leqslant k$. A set is closed in $(b m)_{k}$ topology provided that it is sequentially closed with respect to $(\mathrm{bm})_{k}$-convergence. The topology of bounded convergence in measure on $L^{\infty}(I)=W_{0}^{\infty}(I)$ will be denoted by (bm).

The aim of this paper is to provide nonlinear extensions of the representation theorem described in (0.3). To describe these results it is necessary to introduce some notations and terminology. Let $H$ be a real function on $I \times R$. If $f$ is a real function on $I$ then $\mathbf{H} f$ denotes the function given by $\mathbf{H} f(t)$ $=H(t, f(t)), t \in I$. A function $H$ as above is said to be normalized if $H(\cdot, 0)=0$. Note that if $H$ is normalized and $f, g$ are real functions on $I$ then

$$
\mathbf{H}(f+g)=\mathbf{H} f+\mathbf{H} g \text { provided } f \cdot g=0 .
$$

A function $H: I \times R \rightarrow R$ is called a Carathéodory function if $H(\cdot, a)$ is measurable for every $a$ in $R$ and $H(t, \cdot)$ is continuous for almost every $t$ in $I$. Every Carathéodory function has the property that $\mathbf{H} f$ is measurable whenever $f$ is measurable. For $1 \leqslant p \leqslant \infty$ we denote by $\mathrm{Car}^{p}$ the family of functions $\{H\}$ satisfying:

$$
\left\{\begin{array}{l}
H \text { is Caratheodory function on } I \times R, \\
\mathbf{H} f \in L^{1}(I) \text { whenever } f \in L^{p}(I) .
\end{array} .\right.
$$

Now suppose that $H$ is a normalized function in $\operatorname{Car}^{p}$ and define the functional $M$ on $\stackrel{\circ}{W}_{k}^{p}(I)$ by

$$
M(u)=\int_{I} \mathbf{H}\left(D^{k} u\right) d m, \quad u \in \stackrel{\circ}{W}_{k}^{p}(I) .
$$


It then follows from known results concerning Carathéodory functions (see [7, Chapter $1, \S 2]$ ) that $M$ is continuous on $\mathscr{W}_{k}^{p}(I)$ (and $(b m)_{k}$-continuous, if $p=\infty)$. In addition, it follows from (0.4) that if $u, v \in W_{k}^{p}(I)$ then

$$
M(u+v)=M(u)+M(v) \quad \text { whenever } D^{k} u \cdot D^{k} v=0 .
$$

This property of the functional will be called $D^{k}$-disjoint additivity; in the case $k=0$ it will be called simply disjoint additivity.

Our first main result states that, conversely: Every functional on $\dot{W}_{k}^{p}(I)$ which is continuous $\left((b m)_{k}\right.$-continuous, if $\left.p=\infty\right)$ and $D^{k}$.disjointly additive has a representation of the form (0.6), where $H$ is a normalized function in $\mathrm{Car}^{p}$.

A parallel result holds for operators: If $\mathcal{H}_{:}: \stackrel{\circ}{W}^{p}(I) \rightarrow L^{1}(I)$ is an operator which is continuous $\left((\mathrm{bm})_{k}\right.$-continuous, if $\left.p=\infty\right)$ and $D^{k}$-disjointly additive and if, in addition, $\mathcal{H}$ is "local" in the sense that $\mathcal{H}(u)$ vanishes wherever $D^{k} u$ vanishes, then there exists a normalized function $H$ in $\mathrm{Car}^{p}$ such that

$$
\mathcal{H}(u)=\mathbf{H}\left(D^{k} u\right) \text { for all } u \in \dot{W}_{k}^{p}(I) .
$$

Disjointly additive functionals and operators, defined on various Banach spaces of measurable functions forming a complete lattice, have previously been characterized by several authors. This family of Banach function spaces includes the $L^{p}$ spaces as well as Orlicz spaces. However, it does not include such Banach spaces as Sobolev spaces, and the methods utilized have always depended crucially on the completeness of the lattice structure (see, e.g., [10], [11], [5]). (For the case of transformations on the Bochner spaces of vectorvalued functions $L^{p}(I ; B)$ see [12].) Similar results have been obtained for functionals and operators on spaces of continuous functions which satisfy a notion of additivity somewhat stronger than that of disjoint additivity (see, for example, [4], [2], [1]).

The results which we have stated can be reformulated as representation theorems for disjointly additive functionals (and operators) whose domain is a certain closed subspace of $L^{p}(I)$. In fact this reformulation is essential in our treatment of the representation problem. Thus a natural way of developing the desired representation theorem for functionals would be the following: First extend the given functional to the entire space $L^{p}$, preserving its properties of continuity and disjoint additivity. Then utilize the representation theory for additive functionals on $L^{p}(I)$ which was mentioned above, in order to deduce the representation (0.6). However, in general a disjointly additive, continuous functional on a closed subspace of $L^{p}(I)$ need not have an extension to all of $L^{p}(I)$ which preserves these properties. For instance, one can construct continuous functionals on the subspace consisting of first order polynomials (where every functional is, vacuously, disjointly additive) which do not have a continuous, disjointly additive extension to all of $L^{p}(I)$. This suggests that the 
existence of an extension depends on the specific properties of the closed subspace and of the functional defined on it. Thus, our proof of the existence of an extension is, to a large extent, a by-product of the construction of a kernel representation, rather than an aid in its proof.

A crucial step in proving the existence of a kernel $H$ satisfying (0.6) involves a Radon-Nikodým type theorem for functions whose domain is a subset of $\tau \times \tau$ where $\tau$ denotes the $\sigma$-algebra of Lebesgue sets in $I$.

The plan of the paper is as follows. In $\S 1$ we show that the main results can be reformulated in terms of disjointly additive functionals (resp. operators) on a closed subspace of $L^{p}(I)$, namely, on $\mathfrak{T}_{k}^{p}=\left\{f \in L^{p}(I): \int_{I} f d \mu_{k}=0\right\}$, where $d \mu_{k}=\left(d m, t d m, \ldots, t^{k-1} d m\right)$. In $\S 2$ we discuss algebraic properties of disjointly additive functionals. Some properties of the vector-valued measure $\mu_{k}$ are derived in $\$ 3$. The proof of the main result for disjointly additive functionals is then developed in \$\$4-9. This proof depends on two main auxiliary results. The first is the Radon-Nikodým type theorem mentioned earlier which is proved in $\$ \S 5$ and 6 . The second is a representation theorem for $\mu_{k}$-invariant, disjointly additive functionals, which is given in $\$ 7$. The proof of the main result for disjointly additive operators is developed in $\$ 10$.

1. Statement of main results. We start with some definitions and notations.

Let $H, H^{\prime}$ be two real functions on $I \times R$. We shall say that $H$ and $H^{\prime}$ are equivalent if, for every real $a, H(t, a)=H^{\prime}(t, a)$ a.e. in $I$. If $H$ and $H^{\prime}$ are Carathéodory functions, then they are equivalent if and only if there exists a set $T$ in $\tau$, with $m(T)=1$, such that

$$
H(t, a)=H^{\prime}(t, a), \quad \forall(t, a) \in T \times R .
$$

If $f$ is a real function on $I$ we denote by $H_{f}$ the function with domain $I \times R$ given by

$$
H_{f}(t, a)=a f(t), \quad \forall(t, a) \in I \times R .
$$

Our main results are formulated in the next two theorems.

THEOREM 1.1. Let $M$ be a functional on $\stackrel{\circ}{W}_{k}^{p}(I),(1 \leqslant p \leqslant \infty, k$ a positive integer) possessing the following properties:

(i) $M$ is $D^{k}$-disjointly additive.

(ii) $M$ is continuous with respect to the $W_{k}^{p}$ norm, if $1 \leqslant p<\infty$, and with respect to the $(\mathrm{bm})_{k}$ topology, if $p=\infty$.

Then there exists a normalized function $H: I \times R \rightarrow R$ which belongs to $\mathrm{Car}^{p}$ such that

$$
M(u)=\int_{I} \mathbf{H}\left(D^{k} u\right) d m, \quad \forall u \in \stackrel{W}{k}_{k}^{p}(I)
$$


If $H$ is a normalized Carathéodory function satisfying (1.2) and if $q$ is a polynomial of order less than $\mathrm{k}$, then $\mathrm{H}+\mathrm{H}_{q}$ is also a normalized Carathéodory function and satisfies (1.2). Conversely, if $H$ and $H^{\prime}$ are two normalized Carathéodory functions satisfying (1.2) then there exists a polynomial $q$ of order less than $k$ such that $H^{\prime}-H$ is equivalent to $H_{q}$.

Finally, if $M$ is a functional on $W_{k}^{p}(I)$ defined by (1.2), where $H$ is a normalized function in $\mathrm{Car}^{p}$, then $M$ satisfies conditions (i) and (ii).

THEOREM 1.2. Let $\mathcal{G}: \dot{W}_{k}^{p}(I) \rightarrow L^{1}(I),(1 \leqslant p \leqslant \infty, k$ a positive integer $)$ be an operator satisfying the following conditions:

(i) $\mathcal{G}$ is $D^{k}$-disjointly additive.

(ii) If $1 \leqslant p<\infty, \mathcal{G}$ is continuous with respect to the norm topologies; if $p=\infty, \mathcal{G}$ is continous with respect to the $(\mathrm{bm})_{k}$ topology of $\dot{W}_{k}^{p}(I)$ and the norm topology of $L^{1}(I)$.

(iii) $\mathcal{G}$ is $D^{k}$-local, i.e. for every $u$ in $\dot{W}_{k}^{p}(I), \mathcal{G}(u)$ vanishes whenever $D^{k} u$ vanishes.

Then there exists a normalized function $G: I \times R \rightarrow R$, belonging to $\mathrm{Car}^{p}$ such that

$$
\mathcal{G}(u)=\mathbf{G}\left(D^{k} u\right), \quad \forall u \in \mathscr{W}_{k}^{p}(I) .
$$

The function $G$ is unique up to equivalence.

If S: $W_{k}^{p}(I) \rightarrow L^{1}(I)$ is defined by (1.3), where $G$ is a normalized function in $\mathrm{Car}^{p}$, then $\mathcal{G}$ satisfies condition (i)-(iii).

As we shall show below, these results can be reformulated as representation theorems for functionals and operators defined on a closed subspace of $L^{p}(I)$. It is, actually, in this form that they will be proved here.

Consider the following subspace of $L^{p}(I),(1 \leqslant p \leqslant \infty)$,

$$
\Re_{k}^{p}=\left\{f \in L^{p}(I): \int_{I} t^{j} f(t) d m(t)=0, j=0, \ldots, k-1\right\} .
$$

Clearly $\pi_{k}^{p}$ is a closed subspace of $L^{p}(I)$ with respect to the norm topology. Also $\Re_{k}^{p}$ is closed with respect to the $(\mathrm{bm})$ topology.

It is not difficult to verify that $\Re_{k}^{p}$ is linearly isomorphic with $\mathscr{W}_{k}^{p}(I)$, the isomorphism being given by

$$
\grave{W}_{k}^{p}(I) \ni u \leftrightarrow D^{k} u \in \mathscr{N}_{k}^{p} .
$$

In view of the equivalence of the norms $(0.1)$ and $(0.2)$ the isomorphism is bicontinuous with respect to the norm topologies. If $p=\infty$, the isomorphism is also bicontinuous with respect to the $(\mathrm{bm})_{k}$ and $(\mathrm{bm})$ topologies. Indeed a sequence $\left\{u_{n}\right\}$ in $\dot{W}_{k}^{\infty}(I)$ is $(b m)_{k}$-convergent if and only if $\left\{D^{k} u_{n}\right\}$ is $(b m)$ convergent. The "only if" statement is trivial. The other statement follows 
from the following considerations. If $\left\{D^{k} u_{n}\right\}$ is $(b m)$-convergent, then it is convergent in the $L^{p}$ norm for every $p \in[1, \infty)$. Hence, by the equivalence of (0.1) and (0.2) in $\dot{W}_{k}^{p}(I),\left\{u_{n}\right\}$ is convergent with respect to the $W_{k}^{p}$ norm. Furthermore, since $\left\{D^{k} u_{n}\right\}$ is uniformly bounded, it follows that $\left\{u_{n}\right\}$ is bounded in the $W_{k}^{\infty}$ norm. Thus $\left\{u_{n}\right\}$ is $(b m)_{k}$-convergent.

In view of the isomorphism (1.5), the results stated in the previous two theorems may be reformulated as follows.

THEOREM 1.3. Let $N$ be a functional on $\Re_{k}^{p},(1 \leqslant p \leqslant \infty, k$ a positive integer $)$ satisfying the following conditions.

(i) $N$ is disjointly additive, i.e., if $f, g \in \mathfrak{N}_{k}^{p}$ then

$$
N(f+g)=N(f)+N(g) \text { whenever } f g=0 .
$$

(ii) $N$ is continuous with respect to the $L^{p}$ norm, if $1 \leqslant p \leqslant \infty$, and with respect to the $(\mathrm{bm})$ topology, if $p=\infty$.

Then there exists a normalized function $H$ in $\mathrm{Car}^{p}$ such that

$$
N(f)=\int_{I} \mathbf{H}(f) d m, \quad \forall f \in \mathfrak{N}_{k}^{p} .
$$

If $H$ is a normalized Carathéodory function satisfying (1.6) and $q$ is a polynomial of order less than $k$, then $H+H_{q}$ is a normalized Carathéodory function and satisfies (1.6). Conversely, if $H, H^{\prime}$ are two normalized Carathéodory functions satisfying (1.6), then there exists a polynomial $q$ of order less than $k$, such that $H^{\prime}-H$ is equivalent to $H_{q}$.

Finally, if $N$ is a functional on $\Re_{k}^{p}$ defined by (1.6), where $H$ is a normalized function in $\mathrm{Car}^{p}$, then $N$ satisfies conditions (i) and (ii).

A normalized function in $\mathrm{Car}^{p}$ satisfying (1.6) will be called a Carathéodory kernel for $N$.

THEOREM 1.4. Let $\mathcal{H}$ be an operator, $\mathcal{H}_{:} \mathfrak{N}_{k}^{p} \rightarrow L^{1}(I)$ satisfying the following conditions:

(i) $\mathcal{H}$ is disjointly additive.

(ii) $\mathcal{H}$ is continuous with respect to the $L^{p}$ norm, if $1 \leqslant p<\infty$, and with respect to the (bm) topology, if $p=\infty$.

(iii) $\mathcal{H}$ is local, i.e. for every $f \in \mathfrak{R}_{k}^{p}, \mathcal{H}(f)$ vanishes wherever $f$ vanishes.

Then there exists a normalized function $H: I \times R \rightarrow R$ belonging to $\mathrm{Car}^{p}$ such that

$$
\mathscr{K}(f)=\mathbf{H}(f), \quad \forall f \in \Re_{k}^{p} .
$$

The function $H$ is unique up to equivalence.

If $\mathcal{H}: W_{k}^{p}(I) \rightarrow L^{1}(I)$ is an operator defined by (1.7), where $H$ is a normalized function in $\mathrm{Car}^{p}$, then $\mathcal{H}$ satisfies conditions (i)-(iii). 
The necessity of condition (i) in Theorem 1.3 and of conditions (i) and (iii) in Theorem 1.4 is an immediate consequence of the normalization of $H$. The necessity of the continuity assumption in these two theorems follows from the following known result.

Proposition 1.5. Let $H$ be a Carathéodory function on $I \times R$ and let $p$ $\in[1, \infty]$. Then the following statements are equivalent:

(I) $H \in \mathrm{Car}^{p}$.

(II) If $1 \leqslant p<\infty$, then there exists a function $h$ in $L^{1}(I)$ and a constant $b$ such that

$$
|H(t, a)| \leqslant h(t)+b|a|^{p}, \quad \forall(t, a) \in T \times R,
$$

where $T$ is a subset of $[0,1]$ of total measure (i.e., $m(T)=1)$. If $p=\infty$, then for every positive constant $c$ there exists a function $h_{c}$ in $L^{1}(I)$ such that

$$
|H(t, a)| \leqslant h_{c}(t), \quad \forall(t, a) \in T \times[-c, c]
$$

with $T$ as above.

(III) The operator $\mathcal{H}: L^{p}(I) \rightarrow L^{1}(I)$ given by $\mathcal{H}(f)=\mathbf{H}(f), f \in L^{p}(I)$, is continuous with respect to the norm topologies. If $p=\infty, \mathcal{H}$ is also continuous with respect to the $(\mathrm{bm})$ topology in $L^{\infty}$ and the norm topology in $L^{1}$.

For the proof of this proposition we refer the reader to [7] when $1 \leqslant p<\infty$ and to [8] for $1 \leqslant p \leqslant \infty$. The last statement in (III) does not appear in these references. However, a proof of this statement may be found in [11]. In fact one can easily derive this property from (II) as follows.

Let $\left\{f_{n}\right\}$ be a $(b m)$-convergent sequence in $L^{\infty}(I)$. Then every subsequence $\left\{f_{n_{j}}\right\}$ contains a further subsequence $\left\{f_{n_{j}}\right\}$ which converges to $f$ a.e. Let $c$ be a bound for $\left\{f_{n}\right\}$ and let $h_{c}$ be as in (II) (for $p=\infty$ ). Then $\left\{\mathbf{H}\left(f_{n_{j}}\right)\right\}$ converges a.e. to $\mathbf{H}(f)$ and is dominated by $h_{c}$. Hence, by the dominated convergence theorem, $\mathbf{H}\left(f_{n^{\prime}}\right) \rightarrow \mathbf{H}(f)$ in $L^{1}(I)$. It follows that $\mathbf{H}\left(f_{n}\right) \rightarrow \mathbf{H}(f)$ in $L^{\prime}(I)$.

We finish this section with a remark concerning the normalization of the kernel. If $H$ is a function in $\mathrm{Car}^{p}$, not necessarily normalized, and $N$ is a functional on $\Re_{k}^{p}$ defined by (1.6) then $N$ will satisfy condition (ii) of Theorem 1.3. But, instead of disjoint additivity, we will have

$$
\begin{aligned}
& N(f+g)=N(f)+N(g)-c \\
& \quad \text { whenever } f, g \in \Re_{k}^{p} \text { and } f \cdot g=0 \text {, with } c=\int_{I} H(\cdot, 0) d m .
\end{aligned}
$$

On the other hand, if $N$ is a functional on $\Re_{k}^{p}$ which satisfies (1.8) (with some constant c) and also condition (ii) of Theorem 1.3, then the functional $N^{*}$ defined by

$$
N^{*}(f)=N(f)-c, \quad \forall f \in \mathfrak{T}_{k}^{p}
$$


satisfies all the assumptions of Theorem 1.3. Hence there exists a normalized function $H^{*}$ in $\mathrm{Car}^{p}$ such that

$$
N^{*}(f)=\int_{I} \mathbf{H}^{*}(f) d m, \quad \forall f \in \mathfrak{N}_{k}^{p} .
$$

Furthermore, if $h_{0}$ is any function in $L^{1}(I)$ such that $\int_{I} h_{0} d m=c$ then, setting $H(t, a)=H^{*}(t, a)+h_{0}(t)$ for every $(t, a) \in I \times R$, we have

$$
N(f)=\int_{l} \mathbf{H}(f) d m, \quad \forall f \in \mathscr{N}_{k}^{p} .
$$

2. Some algebraic properties of disjointly additive functionals. All the functions considered below are assumed to be real Lebesgue measurable functions on $I$ and all sets are assumed to belong to $\tau$.

We denote by $\mu_{k}(k=1,2, \ldots)$ the vector-valued measure on $(I, \tau)$ given by

$$
\mu_{k}(E)=\left(\int_{E} d m, \int_{E} t d m, \ldots, \int_{E} t^{k-1} d m\right), \quad \forall E \in \tau
$$

Given an integrable function $f$ we set

$$
\hat{\mu}_{k}(f)=\int_{l} f d \mu_{k} .
$$

Since $\mu_{k}$ is a nonatomic finite dimensional vector measure, Liapunov's theorem [9] gives the following result.

For every set $E$ in $\tau$, there exists a partition $\left\{E_{1}, E_{2}\right\}$ of $E$ such that $\mu_{k}\left(E_{1}\right)=\mu_{k}\left(E_{2}\right)$.

The following two spaces related to $\mu_{k}$ will occur frequently:

$$
\left\{\begin{array}{l}
\Re_{k}^{p}=\left\{f \in L^{p}(I): \hat{\mu}_{k}(f)=0\right\}, \\
\delta_{k}=\left\{f: f \text { is a simple function on } I \text { and } \hat{\mu}_{k}(f)=0\right\} .
\end{array}\right.
$$

Given a function $f$ we shall denote by $K(f)$ the $\operatorname{set}\{t: f(t) \neq 0\} . K(f)$ will be called the strict support of $f$. Two functions are said to be disjoint if their strict supports are disjoint. The characteristic function of a set $E$ will be denoted by $\chi_{E}$.

In the following lemmas we derive certain properties of disjointly additive functionals on $\delta_{k}$, without any continuity assumptions.

LEMMA 2.1. Let $N$ be a disjointly additive, even functional on $\delta_{k}$. Then there exists a family $\left\{\gamma_{a}\right\}_{a \in R}$ of finitely additive set functions on $\tau$ such that

(i) $\gamma_{a}=\gamma_{-a}$ for every real $a$ and $\gamma_{0}=0$;

(ii) if $f \in \mathcal{S}_{k}$ and $f=\sum_{i=1}^{n} \alpha_{i} \chi_{E_{i}}$ with $\left\{E_{i}\right\}$ disjoint, then 


$$
N(f)=\sum_{i=1}^{n} \gamma_{\alpha_{i}}\left(E_{i}\right)
$$

Proof. Let $V$ be a set and let $\left\{V_{1}, V_{2}\right\}$ and $\left\{V_{1}^{\prime}, V_{2}^{\prime}\right\}$ be two partitions of $V$ into sets of equal $\mu_{k}$-measure. We claim that

$$
N\left(a\left(\chi_{V_{1}}-\chi_{V_{2}}\right)\right)=N\left(a\left(\chi_{V_{1}^{\prime}}-\chi_{V_{2}^{\prime}}\right)\right), \quad \forall a \in R .
$$

Set $W_{i, j}=V_{i} \cap V_{j}^{\prime},(i, j=1,2)$. Then $V_{i}=W_{i, 1} \cup W_{i, 2}$ and $V_{j}^{\prime}=W_{1, j}$ $\cup W_{2, j}$. Hence,

$$
\begin{aligned}
& \mu_{k}\left(W_{1,1}\right)+\mu_{k}\left(W_{1,2}\right)=\mu_{k}\left(W_{2,1}\right)+\mu_{k}\left(W_{2,2}\right), \\
& \mu_{k}\left(W_{1,1}\right)+\mu_{k}\left(W_{2,1}\right)=\mu_{k}\left(W_{1,2}\right)+\mu_{k}\left(W_{2,2}\right) .
\end{aligned}
$$

Therefore,

$$
\mu_{k}\left(W_{1,2}\right)=\mu_{k}\left(W_{2,1}\right)
$$

By the disjoint additivity of $N$ and (2.6) we obtain,

$$
\left\{\begin{array}{l}
N\left(a\left(\chi_{V_{1}}-\chi_{V_{2}}\right)\right)=N\left(a\left(\chi_{W_{1,1}}-\chi_{W_{2,2}}\right)\right)+N\left(a\left(\chi_{W_{1,2}}-\chi_{W_{2,1}}\right)\right) \\
N\left(a\left(\chi_{V_{1}^{\prime}}-\chi_{V_{2}^{\prime}}\right)\right)=N\left(a\left(\chi_{W_{1,1}}-\chi_{W_{2,2}}\right)+N\left(a\left(\chi_{W_{2,1}}-\chi_{W_{1,2}}\right)\right)\right.
\end{array}\right.
$$

In view of the fact that $N$ is even, (2.7) yields (2.5).

Given a set $V$ and a real number $a$, let

$$
\gamma_{a}(V)=N\left(a\left(\chi_{V_{1}}-\chi_{V_{2}}\right)\right)
$$

where $\left\{V_{1}, V_{2}\right\}$ is a partition of $V$ into sets of equal $\mu_{k}$-measure. By (2.5), $\gamma_{a}$ is well defined. Clearly $\gamma_{a}$ is a finitely additive set function on $\tau$ and it satisfies condition (i).

Let $f$ be as in (ii) and let $\left\{E_{i, 1}, E_{i, 2}\right\}$ be a partition of $E_{i}$ into sets of equal $\mu_{k}$ measure $(i=1, \ldots, n)$. Set $f_{j}=\sum_{i=1}^{n} \alpha_{i} \chi_{E_{i, j}},(j=1,2)$. Then

$$
\begin{aligned}
N(f) & =N\left(f_{1}\right)+N\left(f_{2}\right)=N\left(f_{1}\right)+N\left(-f_{2}\right)=N\left(f_{1}-f_{2}\right) \\
& =\sum_{i=1}^{n} N\left(\alpha_{i}\left(\chi_{E_{i, 1}}-\chi_{E_{i, 2}}\right)\right)=\sum_{i=1}^{n} \gamma_{\alpha_{i}}\left(E_{i}\right) .
\end{aligned}
$$

This completes the proof of the lemma.

LEMMA 2.2. Let $N$ be a disjointly additive, odd functional on $\delta_{k}$. If $f, g, h$ are disjoint simple functions such that $f-g$ and $f-h$ belong to $\delta_{k}$ then

$$
N(f-g)=N(f-h)-N(g-h) .
$$


Proof. As in the proof of the previous lemma, we assoicate with the given function $f$ a pair of disjoint functions $f_{1}, f_{2}$ such that $f=f_{1}+f_{2}$ and $\hat{\mu}_{k}\left(f_{1}\right)$ $=\hat{\mu}_{k}\left(f_{2}\right)$. Similarly we associate with $g$ and $h$ pairs of functions $g_{1}, g_{2}$ and $h_{1}$, $h_{2}$ respectively. Then

$$
N(f-g)=N\left(f_{1}-g_{1}\right)+N\left(f_{2}-g_{2}\right)+\left[N\left(h_{1}-h_{2}\right)+N\left(h_{2}-h_{1}\right)\right] .
$$

The term in the brackets vanishes because $N$ is odd. Thus

$$
\begin{aligned}
N(f-g) & =N\left(f_{1}-h_{2}\right)+N\left(h_{1}-g_{1}\right)+N\left(f_{2}-h_{1}\right)+N\left(h_{2}-g_{2}\right) \\
& =N(f-h)+N(h-g)=N(f-h)-N(g-h) . .
\end{aligned}
$$

The following is an immediate consequence of the previous two lemmas.

LEMMA 2.3. Let $N$ be a disjointly additive functional on $\delta_{k}$. If $f, g, h$ are disjoint simple functions such that $f-g$ and $f-h$ belong to $\delta_{k}$ then

$$
N(f-h)-N(g-h) \text { is independent of } h \text {. }
$$

Proof. Denote by $N_{\mathrm{o}}$ the odd part of $N$ and by $N_{\mathrm{e}}$ the even part of $N$. Obviously $N_{\mathrm{o}}$ and $N_{\mathrm{e}}$ are disjointly additive on $\delta_{k}$. Applying Lemma 2.1(ii) to $N_{e}$ and Lemma 2.2 to $N_{\mathrm{o}}$ we obtain (2.10).

LEMMA 2.4. Let $U, V$ be sets in $\tau$, not necessarily disjoint, with $\mu_{k}(U)$ $=\mu_{k}(V)$, and let $\left\{U_{\nu}\right\}_{1}^{s},\left\{V_{\nu}\right\}_{1}^{s}$ be partitions of $U, V$ such that

$$
\mu_{k}\left(U_{\nu}\right)=\mu_{k}\left(V_{\nu}\right), \quad \nu=1,2, \ldots, s .
$$

If $N$ is a disjointly additive odd functional on $\varsigma_{k}$ then

$$
\sum_{1}^{s} N\left(\chi_{U_{v}}-\chi_{V_{v}}\right)=N\left(\chi_{U}-\chi_{V}\right)
$$

Proof. Let $W_{\nu, \sigma}=U_{\nu} \cap V_{\sigma}$ and let $\left\{W_{\nu, \sigma}^{1}, W_{\nu, \sigma}^{2}\right\}$ be a partition of $W_{v, \sigma}$ into sets of equal $\mu_{k}$-measure. Set $U_{\nu}^{i}=\cup_{\mu=1}^{s} W_{\nu, \sigma}^{i}, V_{\sigma}^{i}=\cup_{\nu=1}^{s} W_{\nu, \sigma}^{i}, U^{i}=\cup_{\nu=1}^{s} U_{\nu}^{i}$, $V^{i}=\cup_{\sigma=1}^{s} V_{o}^{i},(i=1,2)$. Note that sets with different upper indices are disjoint. By Lemma 2.2

$$
N\left(\chi_{U^{1}}-\chi_{V^{1}}\right)=N\left(\chi_{U^{1}}-\chi_{U^{2}}\right)-N\left(\chi_{V^{1}}-\chi_{U^{2}}\right) .
$$

Actually Lemma 2.2 gives this result in the case where $U^{1}, V^{1}, U^{2}$ are disjoint. But it is easily seen that the equality holds even if $U^{1}, V^{1}$ are not disjoint. Further, by disjoint additivity,

$$
N\left(\chi_{U^{1}}-\chi_{U^{2}}\right)=\sum_{\nu=1}^{s} N\left(\chi_{U_{\nu}^{1}}-\chi_{U_{\nu}^{2}}\right)
$$


and

$$
N\left(\chi_{V^{1}}-\chi_{U^{2}}\right)=\sum_{\nu=1}^{s} N\left(\chi_{V_{\nu}^{1}}-\chi_{U_{\nu}^{2}}\right)
$$

Thus, using Lemma 2.2 once more,

$$
N\left(\chi_{U^{1}}-\chi_{V^{1}}\right)=\sum_{\nu=1}^{s}\left[N\left(\chi_{U_{\nu}^{\prime}}-\chi_{U_{\nu}^{2}}\right)-N\left(\chi_{V_{\nu}^{1}}-\chi_{U_{\nu}^{2}}\right)\right]=\sum_{\nu=1}^{s} N\left(\chi_{U_{\nu}^{\prime}}-\chi_{V_{\nu}^{1}}\right) .
$$

Similarly,

$$
N\left(\chi_{U^{2}}-\chi_{V^{2}}\right)=\sum_{\nu=1}^{s} N\left(\chi_{U_{\nu}^{2}}-\chi_{V_{\nu}^{2}}\right)
$$

Combining the last two equations we obtain (2.12).

COROLlaRY 2.5. Under the assumptions of the lemma we have

$$
\sum_{1 \leqslant \nu, \sigma \leqslant s} N\left(\chi_{U_{\nu}}-\chi_{V_{o}}\right)=s N\left(\chi_{U}-\chi_{V}\right)
$$

Proof. This is an immediate consequence of (2.12).

REMarK. Suppose that in addition to the assumption of Lemma 2.4, $N$ is countably disjointly additive. Then Lemma 2.4 holds if finite partitions are replaced by countable partitions. The proof is the same as before.

3. Some properties of $\mu_{k}$. For later developments we need more detailed information concerning the measure $\mu_{k}$. This additional information will be derived in the present section.

Given a set $E$ in $\tau$ we shall say that $\Sigma_{E}$ is a Blackwell $\sigma$-algebra for $E$ with respect to $\mu_{k}$ if,

(i) $\Sigma_{E}$ is a $\sigma$-algebra for $E$ and $\Sigma_{E} \subseteq \tau$.

(ii) $F \in \Sigma_{E} \Rightarrow \mu_{k}(F)$ is parallel to $\mu_{k}(E)$.

(iii) $\mu_{k}$ is a nonatomic measure on $\Sigma_{E}$. (Since the range of $\mu_{k}$ on $\Sigma_{E}$ is onedimensional, nonatomicity can be understood just as in the case of scalar measures.)

It is well known that every set $E$ possesses such a $\sigma$-algebra. In fact this is a consequence of Liapunov's theorem on vector measures [9] (see also [3] which first discussed such algebras). Moreover by Liapunov's theorem, the range of $\mu_{k}$ on $\Sigma_{E}$ is the segment $\left\{a \mu_{k}(E): 0 \leqslant a \leqslant 1\right\}$.

Definition 3.1. Given a family of disjoint sets $\mathbb{Q}=\left\{A_{1}, \ldots, A_{k}\right\}$, we shall say that it is $\mu_{k}$-independent if $\left\{\mu_{k}\left(A_{j}\right)\right\}_{j=1}^{k}$ is a linearly independent set of vectors.

Let $\mathcal{Q}$ be a $\mu_{k}$-independent family of sets. We denote by $P_{Q}: R_{k} \rightarrow R_{k}$ the linear mapping given by 


$$
P_{\mathbb{Q}}(x)=\left(\sigma_{1}, \ldots, \sigma_{k}\right) \quad \text { where } x=\sum_{j=1}^{k} \sigma_{j} \mu_{k}\left(A_{j}\right) .
$$

The norm of $P_{\mathbb{Q}}$ with respect to the max norm in $R_{k}$ will be denoted by $1 / \rho(\mathbb{Q})$.

If $T$ is a set, then $P_{\mathbb{Q}}\left(\mu_{k}(T)\right)$ will be abbreviated to $P_{\mathscr{Q}}(T)$.

Lemma 3.1. Let $k$ be a positive integer and let $\alpha \in(0,1]$. Then there exists a positive number $\rho_{k}(\alpha)$ such that the following statement holds.

For every set $D$ of measure $\alpha$, there exists a $\mu_{k}$-independent family $\&$ consisting of subsets of $D$, such that $\rho(\mathbb{Q}) \geqslant \rho_{k}(\alpha)$.

Proof. Denote by $p\left(t_{1,1}, \ldots, t_{k-1, k}\right)$ the determinant of the $k \times k$ matrix whose $j$ th column is given by $\left(1, t_{1, j}, t_{2, j}^{2}, \ldots, t_{k-1, j}^{k-1}\right)$. Let $p_{0}\left(t_{1}, \ldots, t_{k}\right)$ be the Van der Monde determinant whose $j$ th column is given by $\left(1, t_{j}, t_{j}^{2}, \ldots, t_{j}^{k-1}\right)$. If $\beta>0$, and $t_{j+1}-t_{j} \geqslant \beta$ for $j=1, \ldots, k-1$ then

$$
p_{0}\left(t_{1}, \ldots, t_{k}\right) \geqslant \beta^{k(k-1) / 2}=\nu(\beta, k) .
$$

Let $\delta=\delta(\beta, k)$ be a positive number such that

$$
\left.\begin{array}{c}
\left\{t_{1}, \ldots, t_{k}\right\} \subseteq[0,1], \\
t_{j+1}-t_{j} \geqslant \beta, j=1, \ldots, k-1, \\
\left|t_{i, j}-t_{j}\right| \leqslant \delta, i=1, \ldots, k, \\
j=1, \ldots, k
\end{array}\right\} \Rightarrow p\left(t_{1,1}, \ldots, t_{k-1, k}\right) \geqslant \nu(\beta, k) / 2 .
$$

The existence of such a $\delta$ follows from the continuity of the determinant as a function of its entries.

Let $D$ be a set of measure $\alpha$ and set $\beta=\alpha / 2 k$. Let $\left\{I_{j}=\left[t_{j}^{\prime}, t_{j}^{\prime \prime}\right]\right\}_{1}^{k}$ be a family of intervals such that

(i) $m\left(D \cap I_{j}\right)=\beta, j=1, \ldots, k$;

(ii) $t_{j+1}^{\prime}-t_{j}^{\prime \prime}=\beta, j=1, \ldots, k-1$.

Such a family may be obtained by constructing $I_{1}, \ldots, I_{k}$ in their order of succession, taking $t_{1}^{\prime}=0$. Let $\delta_{0}=\min \left(\frac{1}{2} \delta(\beta, k), \beta\right)$ and pick $\delta_{1}, \ldots, \delta_{k}$ in the interval $\left[\delta_{0}, 2 \delta_{0}\right]$ such that $m\left(I_{j}\right) / \delta_{j}$ is an integer $(j=1, \ldots, k)$. Consider the partition of $I_{j}$ into subintervals of length $\delta_{j}$. There exists at least one interval in this partition, say $I_{j}^{\prime}$, such that

(iii) $m\left(D \cap I_{j}^{\prime}\right) / \delta_{j} \geqslant m\left(D \cap I_{j}\right) / m\left(I_{j}\right) \geqslant \beta, j=1, \ldots, k$.

Set $A_{j}=D \cap I_{j}^{\prime}$ and $\mathbb{Q}=\left\{A_{1}, \ldots, A_{k}\right\}$. Let

$$
t_{i, j}^{i}=\frac{1}{m\left(A_{j}\right)} \int_{A_{j}} t^{i} d m, \quad i=0, \ldots, k-1 ; j=1, \ldots, k .
$$

Let $t_{j}$ be the left endpoint of $I_{j}^{\prime}$. Then $t_{j+1}-t_{j} \geqslant \beta$ and $0 \leqslant t_{i, j}-t_{j} \leqslant \delta_{j}$ $\leqslant \delta(\beta, k)$ for all $i, j$. Hence, by $(3.2)$, 


$$
p\left(t_{1,1}, \ldots, t_{k-1, k}\right) \geqslant \nu(\beta, k) / 2 .
$$

Let $\Delta=\Delta(\mathbb{Q})$ be the determinant whose columns are given by $\mu_{k}\left(A_{1}\right), \ldots$, $\mu_{k}\left(A_{k}\right)$. Then by (iii) and (3.3) we have,

$$
\begin{aligned}
\Delta(\mathfrak{Q}) & =\left(\prod_{1}^{k} m\left(A_{j}\right)\right) p\left(t_{1,1}, \ldots, t_{k-1, k}\right) \\
& \geqslant\left[\prod_{1}^{k}\left(\beta \delta_{j}\right)\right] \nu(\beta, k) / 2 \geqslant\left(\beta \delta_{0}\right)^{k} \nu(\beta, k) / 2=\nu^{\prime}(\alpha, k) .
\end{aligned}
$$

Thus $\mathbb{Q}$ is $\mu_{k}$-independent.

If $x \in R_{k}$ and $x=\sum_{1}^{k} \sigma_{j} \mu_{k}\left(A_{j}\right)$ then

$$
\left|\sigma_{j}\right| \leqslant c_{k}|x|^{\prime} / \Delta(\mathbb{Q})
$$

where $c_{k}$ is a constant depending only on $k$ (we may take $c_{k}=k^{(k+1) / 2}$ ) and ||$^{\prime}$ denotes the max norm in $R_{k}$. Setting $\rho=\nu^{\prime}(\alpha, k) / c_{k}$ we get, from (3.4) and (3.5), $\left|P_{\mathscr{Q}}(x)\right|^{\prime} \leqslant \rho^{-1}|x|^{\prime}$. This completes the proof of the lemma.

REMARK. Using the notation of Definition 3.1 we have

$$
\left|P_{\mathscr{Q}}(x)\right|^{\prime} \leqslant \rho(\mathbb{Q})^{-1}|x|^{\prime}, \quad \forall x \in R_{k} .
$$

On the other hand, it is easily verified that

$$
|x|^{\prime} \leqslant\left(\sum_{1}^{k} m\left(A_{j}\right)\right)\left|P_{Q}(x)\right|^{\prime} .
$$

Hence, $\rho(\mathbb{Q}) \leqslant \sum_{1}^{k} m\left(A_{j}\right)$. In particular, $\rho_{k}(\alpha) \leqslant \alpha$.

Definition 3.2. Let $\mathbb{Q}=\left\{A_{1}, \ldots, A_{k}\right\}$ be a $\mu_{k}$-independent family of sets. Let $\Sigma_{j}$ be a Blackwell $\sigma$-algebra for $A_{j}$ and set

$$
\Sigma=\Sigma(\mathscr{Q})=\left\{\bigcup_{1}^{k} V_{j}: V_{j} \in \Sigma_{j}, j=1, \ldots, k\right\} .
$$

Let $x=\left(x_{1}, \ldots, x_{k}\right) \in R_{k}$. A function $h$ will be called a $\Sigma$-representative of $x$ if the following conditions are satisfied:

$$
\left\{\begin{array}{l}
h=\chi_{U}-\chi_{V}, U \cap V=\varnothing, U, V \in \Sigma, \\
x=\hat{\mu}_{k}(h)=\mu_{k}(U)-\mu_{k}(V) .
\end{array}\right.
$$

If $T$ is in $\tau$ then a $\Sigma$-representative of $\mu_{k}(T)$ will also be referred to as a $\Sigma$ representative of $T$.

Let $f$ be a simple function: $f=\sum_{i=1}^{n} \alpha_{i} \chi_{E_{i}}$, with $\left\{E_{i}\right\}$ disjoint. We shall say that $\tilde{f}$ is a $\Sigma$-representative of $f$ if $\tilde{f}=\sum_{1}^{n} \alpha_{i} h_{i}$ where $h_{i}$ is a $\Sigma$-representative of $E_{i}$ and $K\left(h_{1}\right), \ldots, K\left(h_{n}\right)$ are disjoint. 
Lemma 3.2. Let $\mathbb{Q}$ and $\Sigma$ be as in the above definition. If $x=\left(x_{1}, \ldots, x_{k}\right)$ $\in R_{k}$ and $|x|^{\prime}=\max _{1 \leqslant j \leqslant k}\left|x_{j}\right| \leqslant \rho(\mathbb{Q})$ then there exists a $\Sigma$-representative of $x$, say $h$, such that

$$
m(K(h)) \leqslant\left[\sum_{1}^{k} m\left(A_{j}\right) / \rho(\mathbb{Q})\right]|x|^{\prime} \leqslant \rho(\mathbb{Q})^{-1}|x|^{\prime} .
$$

Proof. By definition $\left|P_{\mathscr{Q}}(x)\right|^{\prime} \leqslant \rho(\mathfrak{Q})^{-1}|x|^{\prime}$. Hence, if $P_{\mathscr{Q}}(x)=\left(\sigma_{1}, \ldots, \sigma_{k}\right)$, we have $\left|\sigma_{j}\right| \leqslant 1,(j=1, \ldots, k)$, and

$$
\sum_{1}^{k}\left|\sigma_{j}\right| m\left(A_{j}\right) \leqslant\left[\rho(\mathbb{Q})^{-1} \sum_{1}^{k} m\left(A_{j}\right)\right]|x|^{\prime} .
$$

Let $W_{j}$ be a set in $\Sigma_{j}$ such that $m\left(W_{j}\right)=\left|\sigma_{j}\right| m\left(A_{j}\right)$. Then the function $h$ given by

$$
h=\sum_{1}^{k} \operatorname{sign}\left(\sigma_{j}\right) \chi_{W_{j}}
$$

satisfies conditions (3.7). Indeed, $h$ may be written in the form $\chi_{U}-\chi_{V}$ with $U$ and $V$ disjoint sets in $\Sigma$, and

$$
\hat{\mu}_{k}(h)=\sum_{1}^{k}\left(\operatorname{sign} \sigma_{j}\right) \mu_{k}\left(W_{j}\right)=\sum_{1}^{k} \sigma_{j} \mu_{k}\left(A_{j}\right)=x .
$$

Corollary 3.3. If $\left\{T_{1}, \ldots, T_{l}\right\}$ is a collection of sets such that $\Sigma_{1}^{l} m\left(T_{i}\right)$ $\leqslant \rho(\mathbb{Q})$ then there exist disjoint functions $h_{1}, \ldots, h_{l}$ such that $h_{i}$ is a $\Sigma$ representative of $T_{i}$ and

$$
m\left(K\left(h_{i}\right)\right) \leqslant\left[\rho(\mathscr{Q})^{-1} \sum_{1}^{k} m\left(A_{j}\right)\right] m\left(T_{i}\right), \quad(i=1, \ldots, l) .
$$

If $f$ is a simple function such that $m(K(f)) \leqslant \rho(\mathbb{Q})$, then there exists a $\Sigma$ representative of $f$.

Proof. This is an immediate consequence of the lemma and the construction described in its proof.

Lemma 3.4. Let $A$ and $\Sigma$ be as in Definition 3.2. Let $f$ be a simple function and suppose that there exists a $\Sigma$-representative of $f$ which we denote by $\tilde{f}$. Set $f=\sum_{1}^{k} g_{j}$ where $g_{j}$ is a simple function on $\Sigma_{j},(j=1, \ldots, k)$.

If $f \in \delta_{k}$ then $g_{j} \in \delta_{k}$ for all $j$. If for a given number $a$,

$$
\mu_{k}\left(f^{-1}(a)\right)=\mu_{k}\left(f^{-1}(-a)\right)
$$

then

$$
\mu_{k}\left(g_{j}^{-1}(a)\right)=\mu_{k}\left(g_{j}^{-1}(-a)\right), \quad(j=1, \ldots, k) .
$$


Proof. If $f \in \delta_{k}$ then $\tilde{f} \in \delta_{k}$. Thus $\Sigma_{1}^{k} \hat{\mu}_{k}\left(g_{j}\right)=0$. However, $\hat{\mu}_{k}\left(g_{j}\right)$ is parallel to $\mu_{k}\left(A_{j}\right), j=1, \ldots, k$, and $\left\{\mu_{k}\left(A_{j}\right)\right\}_{1}^{k}$ is linearly independent. Hence $\hat{\mu}_{k}\left(g_{j}\right)=0$ for all $j$.

As a consequence of Definition 3.2

$$
\mu_{k}\left(f^{-1}(a)\right)-\mu_{k}\left(f^{-1}(-a)\right)=\mu_{k}\left(\tilde{f}^{-1}(a)\right)-\mu_{k}\left(\tilde{f}^{-1}(-a)\right), \quad \forall a \in R .
$$

Since $g_{1}, \ldots, g_{k}$ are disjoint,

$$
\mu_{k}\left(\tilde{f}^{-1}(a)\right)-\mu_{k}\left(\tilde{f}^{-1}(-a)\right)=\sum_{1}^{k}\left[\mu_{k}\left(g_{j}^{-1}(a)\right)-\mu_{k}\left(g_{j}^{-1}(-a)\right)\right] .
$$

If $a$ is a number such that (3.12) holds, (3.14) and (3.15) imply that

$$
\sum_{1}^{k}\left[\mu_{k}\left(g_{j}^{-1}(a)\right)-\mu_{k}\left(g_{j}^{-1}(-a)\right)\right]=0 .
$$

In view of the linear independence of $\left\{\mu_{k}\left(A_{j}\right)\right\}_{1}^{k},(3.16)$ implies (3.13).

We conclude this section with some more definitions and notations.

Given a set $F$ and a positive integer $r$, there exist partitions of $F$ consisting of $r$ sets of equal $\mu_{k}$ measure. Such a partition may be constructed from subsets of any Blackwell $\sigma$-algebra for $F$. Hence, given a simple function $f$ there exist simple functions $f_{1}, \ldots, f_{r}$ such that

$$
\left\{\begin{array}{l}
\left\{f_{i}^{-1}(a)\right\}_{1}^{r} \text { is a partition of } f^{-1}(a), \\
\mu_{k}\left(f_{i}^{-1}(a)\right)=\frac{1}{r} \mu_{k}\left(f^{-1}(a)\right), \quad(i=1, \ldots, r), \quad \forall a \in R .
\end{array}\right.
$$

A collection of functions $\left\{f_{1}, \ldots, f_{r}\right\}$ satisfying (3.17) will be called a $\mu_{k}$-uniform decomposition of $f$.

Let $E$ be in $\tau$ with $m(E)>0$ and let $\Sigma_{E}$ be a Blackwell $\sigma$-algebra for $E$ with respect to $\mu_{k}$. We denote by $\Re_{k}^{p}\left(\Sigma_{E}\right)$ and $\delta_{k}\left(\Sigma_{E}\right)$ the subsets of $\mathfrak{\pi}_{k}^{p}$ and $\delta_{k}$ respectively, consisting of those functions which are measurable with respect to $\Sigma_{E}$.

4. Statement and discussion of intermediate results. The proof of Theorem 1.3 is divided into two main parts. In the first part we establish the existence of a kernel $H$ (not necessarily Carathéodory) such that the representation formula (1.6) holds with respect to functions in $\delta_{k}$. In the second part we derive certain continuity properties of the kernel mentioned above and use this information to obtain a continuous, disjointly additive extension of the functional $N$ to the entire space $L^{P}(I)$. As we remarked in the introduction, once the existence of such an extension is established, the full representation theorem follows from known results such as those developed in [5]. 
Thus the first part of our program consists in proving the following result.

THEOREM 4.1. Let $N$ be a functional on $\delta_{k}$ satisfying the following conditions.

(i) $N$ is disjointly additive.

(ii) $N$ is continuous with respect to the (bm) topology.

Then there exists a function $H: I \times R \rightarrow R$ such that

$$
\left\{\begin{array}{l}
H(\cdot, a) \in L^{1}(I), \quad \forall a \in R, \\
H(\cdot, 0)=0,
\end{array}\right.
$$

and

$$
N(f)=\int_{I} \mathbf{H}(f) d m, \quad \forall f \in \delta_{k} .
$$

If $q$ is a polynomial of order less than $k$, then $H+H_{q}$ also satisfies (4.1) and (4.2). Conversely, if $H, H^{\prime}$ are two real functions on $I \times R$ satisfying (4.1) and (4.2) then there exists a polynomial $q$ of order less than $k$ such that $H^{\prime}-H$ is equivalent to $H_{q}$.

A function $H$ satisfying (4.1) and (4.2) will be called a kernel for $N$.

In order to prove this theorem, it is convenient to consider odd and even functionals separately. When $N$ is even the existence of a kernel is easily established. We deal with this case in the next lemma. However when $N$ is odd the proof of the theorem is considerably more involved. This part of the proof will be developed in the next three sections.

LEMMA 4.2. Suppose that $N$ is an even functional on $\delta_{k}$ satisfying conditions (i) and (ii) of Theorem 4.1. Then there exists a kernel for $N$.

Proof. Let $\left\{\gamma_{a}\right\}$ be as in Lemma 2.1. Then conditions (i) and (ii) imply that, for every $a$ in $R, \gamma_{a}$ is countably additive on $\tau$ and $\gamma_{a}$ is absolutely continuous with respect to Lebesgue measure. Hence, by the Radon-Nikodým theorem, there exists a function $H: I \times R \rightarrow R$ satisfying (4.1) such that

$$
\gamma_{a}(V)=\int_{V} H(t, a) d m, \quad \forall V \in \tau .
$$

(Since $\gamma_{a}=\gamma_{-a}$ we have also, $H(\cdot, a)=H(\cdot,-a)$, for all $a$ in $R$.)

Now formula (4.2) follows immediately from (4.1), (4.3) and Lemma 2.1(ii).

The next lemma shows (for general $N$ ) that, in order to obtain the representation formula (4.2) for every $f$ in $\delta_{k}$, it is sufficient to establish this formula for a more restricted set of functions.

At this point it is convenient to define two subsets of $\delta_{k}$, namely

$$
\left\{\begin{array}{l}
\mathcal{E}_{k}=\left\{f \in \delta_{k}: \operatorname{range}(f) \subseteq\{0, a, b\} \text { for some } a, b \text { in } R\right\}, \\
\mathcal{E}_{k}^{0}=\left\{f \in \delta_{k}: \operatorname{range}(f) \subseteq\{0,1,-1\}\right\} .
\end{array}\right.
$$


LEMMA 4.3. Let $N$ be a disjointly additive functional on $\delta_{k}$. Suppose that there exists a function $H: I \times R \rightarrow R$, satisfying (4.1), such that

$$
N(f)=\int_{I} \mathbf{H}(f) d m
$$

for every $f$ in $\mathcal{E}_{k}$.

Then (4.5) holds for every $f$ in $\varsigma_{k}$.

Proof. First suppose that $k=1$. In this case it can be verified that every function $f$ in $\delta_{1}$ can be written as a sum of disjoint functions in $\mathcal{E}_{1}$. Therefore the required result follows immediately by disjoint additivity.

Now consider the case $k>1$. In order to prove the lemma it is sufficient to show that (4.5) holds for every $f$ in $\delta_{k}$ such that

$$
m(K(f)) \leqslant \rho_{k}\left(\frac{1}{2}\right),
$$

with $\rho_{k}(\alpha)$ as in Lemma 3.1. Indeed, by means of $\mu_{k}$-uniform decompositions, every function in $\delta_{k}$ can be written as a disjoint sum of functions in $\delta_{k}$ which satisfiy (4.6).

Let $f$ be a function in $\delta_{k}$ satisfying (4.6) and set $D=I \backslash K(f)$. Since $\rho_{k}(\alpha) \leqslant \alpha$ we have $m(D) \geqslant \frac{1}{2}$. Let $\mathcal{Q}$ be a family of $\mu_{k}$-independent subsets of $D$ such that $\rho(\mathbb{Q}) \geqslant \rho_{k}\left(\frac{1}{2}\right)$. The existence of such a family is guaranteed by Lemma 3.1. Let $\Sigma_{j}(j=1, \ldots, k)$ and $\Sigma$ be as in Definition 3.2. Finally, let $\tilde{f}$ be a $\Sigma$-representative of $-f$. Then $f+\tilde{f}$ is a linear combination of disjoint functions in $\mathcal{E}_{k}^{0}$, so that by $(4.5)$ and disjoint additivity,

$$
N(f)+N(\tilde{f})=N(f+\tilde{f})=\int_{I} \mathbf{H}(f+\tilde{f}) d m .
$$

Let $\tilde{f}=\Sigma_{1}^{k} g_{j}$, where $g_{j}$ is a simple function over $\Sigma_{j}$. By Lemma 3.4, $g_{j} \in \delta_{k}\left(\Sigma_{j}\right)$. Since $\Sigma_{j}$ is a Blackwell $\sigma$-algebra, it can be verified (exactly as in the case $k=1)$ that every function in $\delta_{k}\left(\Sigma_{j}\right)$ can be written as a sum of disjoint functions in $\varepsilon_{k}\left(\Sigma_{j}\right)$. Therefore $\tilde{f}$ can be written as a sum of disjoint functions in $\mathcal{E}_{k}$ and we conclude that

$$
N(\tilde{f})=\int_{I} \mathbf{H}(\tilde{f}) d m .
$$

Finally (4.7) and (4.8) imply (4.5).

The proof of Theorem 4.1 in the case that $N$ is odd is divided into two parts. In the first part we obtain a representation formula for $N$ on the family of functions $\left\{a f: f \in \mathcal{E}_{k}^{0}, a \in R\right\}$. Employing this result we reduce the representation problem to the case of a functional $\hat{N}$ which possesses the additional property of $\mu_{k}$-invariance. We say that a functional $\hat{N}$ on $\delta_{k}$ is $\mu_{k}$-invariant if for every pair of functions $f, g$ in $\delta_{k}$ such that $\mu_{k}\left(f^{-1}(a)\right)=\mu_{k}\left(g^{-1}(a)\right), \forall a$ 
$\in R$ we have $\hat{N}(f)=\hat{N}(g)$. The second part of the proof consists of the derivation of a kernel representation for $\mu_{k}$-invariant functionals.

The representation formula to be obtained in the first part is a consequence of the following result.

THEOREM 4.4. Let $\Lambda$ be a functional on $\varepsilon_{k}^{0}$ satisfying the following conditions:

(i) $\Lambda$ is disjointly additive.

(ii) $\Lambda$ is odd.

(iii) $\Lambda$ is continuous on $\mathcal{E}_{k}^{0}$ with respect to convergence in measure.

Then, there exists a unique $G$ in $L^{1}(I)$ such that $\hat{\mu}_{k}(G)=0$ and,

$$
\Lambda(f)=\int_{I} G f d m, \quad \forall f \in \mathcal{E}_{k}^{0} .
$$

Theorem 4.4 will be proved in $\S 5$ (for $k=1$ ) and in $\S 6$ (for $k>1$ ). The representation formula for $\mu_{k}$-invariant functionals will be derived in $\$ 7$. Finally, the proof of Theorem 4.1 will be completed in $\$ 8$.

5. Proof of Theorem 4.4 for $k=1$. The theorem will be proved by constructing a signed measure $\Lambda_{0}$ on $(I, \tau)$ such that $\Lambda_{0}$ is absolutely continuous with respect to $m$ and

$$
\Lambda\left(\chi_{U}-\chi_{V}\right)=\Lambda_{0}(U)-\Lambda_{0}(V)
$$

whenever $U, V \in \tau$ and $m(U)=m(V) . \Lambda_{0}$ will be constructed in three main steps.

We start with a remark. If $U, V, T$ are sets in $\tau$, of equal $\mu_{k}$ measure, and if $U \cap T=V \cap T=\varnothing$ then,

$$
\Lambda\left(\chi_{U}-\chi_{V}\right)=\Lambda\left(\chi_{U}-\chi_{T}\right)-\Lambda\left(\chi_{V}-\chi_{T}\right)
$$

When $U, V, T$ are disjoint this is proved exactly as Lemma 2.2. The case where $U \cap V \neq \varnothing$ is easily reduced to the preceding one.

Lemma 2.4 remains valid if $N$ is defined only on $\varepsilon_{k}^{0}$. Therefore it may be applied to $\Lambda$. Properties (i) and (iii) of $\Lambda$ imply that $\Lambda$ is countably disjointly additive. Therefore, in this case, Lemma 2.4 holds also if finite partitions are replaced by countable partitions.

STEP 1. Let $U$ be a set in $\tau$ such that $m(U)=1 / n$, where $n$ is an integer larger than 1. Let $\left\{U_{1}, \ldots, U_{n-1}\right\}$ be a partition of $I \backslash U$ into sets of measure $1 / n$ and set

$$
\Lambda_{0}(U)=\frac{1}{n} \sum_{1}^{n-1} \Lambda\left(\chi_{U}-\chi_{U_{i}}\right)
$$

The definition of $\Lambda_{0}(U)$ does not depend on the partition. Indeed, if $\left\{U_{1}^{\prime}, \ldots, U_{n-1}^{\prime}\right\}$ is another partition of $I \backslash U$ into sets of measure $1 / n$ we have 


$$
\sum_{i=1}^{n-1}\left[\Lambda\left(\chi_{U}-\chi_{U_{i}}\right)-\Lambda\left(\chi_{U}-\chi_{U_{i}^{\prime}}\right)\right]=\sum_{i=1}^{n-1} \Lambda\left(\chi_{U_{i}^{\prime}}-\chi_{U_{i}}\right)=0
$$

by (5.1) and Lemma 2.4 .

LEMMA 5.1. (a) If $U$ is a set in $\tau$ with $m(U)=1 / n$ and if $\left\{U^{1}, \ldots, U^{s}\right\}$ is a partition of $U$ into sets of equal measure, then

$$
\Lambda_{0}(U)=\sum_{1}^{s} \Lambda_{0}\left(U^{\nu}\right)
$$

(b) If $U, V$ are sets in $\tau$ such that $m(U)=m(V)=1 / n$, then

$$
\Lambda\left(\chi_{U}-\chi_{V}\right)=\Lambda_{0}(U)-\Lambda_{0}(V) .
$$

Proof. (a) Let $\left\{W_{j}\right\}_{1}^{n-1}$ be a partition of $I \backslash U$ into sets of equal measure. Let $\left\{W_{j}^{i}\right\}_{i=1}^{s}$ be a partition of $W_{j}$ into sets of equal measure. Finally, set $W_{n}^{i}$ $=U^{i}(i=1, \ldots, s)$ and $W_{n}=U$. Then, by (5.2)

$$
\Lambda_{0}\left(U^{\nu}\right)=\frac{1}{n s} \sum_{i=1}^{s} \sum_{j=1}^{n} \Lambda\left(\chi_{U^{\nu}}-\chi_{W_{j}}\right)
$$

On the other hand, by Corollary 2.5 ,

$$
\Lambda\left(\chi_{U}-\chi_{W_{j}}\right)=\frac{1}{s} \sum_{1 \leqslant i, \nu \leqslant s} \Lambda\left(\chi_{U^{v}}-\chi_{W_{j}^{j}}\right) \quad(j=1, \ldots, n) .
$$

Thus,

$$
\Lambda_{0}(U)=\frac{1}{n} \sum_{j=1}^{n} \Lambda\left(\chi_{U}-\chi_{W_{j}}\right)=\frac{1}{n s} \sum_{j=1}^{n} \sum_{1 \leqslant i, \nu \leqslant s} \Lambda\left(\chi_{U^{r}}-\chi_{W_{j}}\right) .
$$

Equalities (5.5) and (5.6) imply (5.3).

(b) First suppose that $U \cap V=\varnothing$. Let $\left\{W_{1}, \ldots, W_{n-2}\right\}$ be a partition of $I \backslash(U \cup V)$ into sets of equal measure. Then,

$$
\begin{aligned}
\Lambda_{0}(U) & -\Lambda_{0}(V) \\
= & \frac{1}{n}\left[\Lambda\left(\chi_{U}-\chi_{V}\right)+\sum_{1}^{n-2} \Lambda\left(\chi_{U}-\chi_{W_{i}}\right)-\Lambda\left(\chi_{V}-\chi_{U}\right)-\sum_{1}^{n-2} \Lambda\left(\chi_{V}-\chi_{W_{i}}\right)\right] \\
= & \Lambda\left(\chi_{U}-\chi_{V}\right) .
\end{aligned}
$$

Here we used (5.1) and the fact that $\Lambda$ is odd.

Now consider the general case. Let $\bar{U}=U \backslash V, \bar{V}=V \backslash U$ and $W=U$ $\cap V$. Let $\left\{\bar{U}_{1}, \bar{U}_{2}\right\},\left\{\bar{V}_{1}, \bar{V}_{2}\right\},\left\{W_{1}, W_{2}\right\}$ be partitions of $\bar{U}, \bar{V}, W$ respectively into sets of equal measure. Set $U_{i}=\bar{U}_{i} \cup W_{i}, V_{i}=\bar{V}_{i} \cup W_{i}(i=1,2)$. Then, by 
the preceding part of the proof we have

$$
\begin{gathered}
\Lambda_{0}\left(U_{1}\right)-\Lambda_{0}\left(U_{2}\right)=\Lambda\left(\chi_{U_{1}}-\chi_{U_{2}}\right), \\
\Lambda_{0}\left(V_{1}\right)-\Lambda_{0}\left(U_{2}\right)=\Lambda\left(\chi_{V_{1}}-\chi_{U_{2}}\right) .
\end{gathered}
$$

Combining the two equalities and using (5.1) we get

$$
\Lambda_{0}\left(U_{1}\right)-\Lambda_{0}\left(V_{1}\right)=\Lambda\left(\chi_{U_{1}}-\chi_{V_{1}}\right)
$$

Similarly we get

$$
\Lambda_{0}\left(U_{2}\right)-\Lambda_{0}\left(V_{2}\right)=\Lambda\left(\chi_{U_{2}}-\chi_{V_{2}}\right) .
$$

In view of the disjoint additivity of $\Lambda$ and part (a) of the lemma, (5.7) and (5.8) imply (5.4).

We conclude the first step of the construction by defining

$$
\Lambda_{0}(U)=0 \text { whenever } U \in \tau \text { and } m(U)=1 \text { or } m(U)=0 .
$$

With this definition, Lemma 5.1(a) remains valid in the case $m(U)=1$. This follows from Corollary 2.5 together with (5.2).

STEP 2. Let $U$ be a set in $\tau$ such that $m(U)$ is rational. Let $m(U)=l / n(l, n$ positive integers) and let $\left\{U_{1}, \ldots, U_{l}\right\}$ be a partition of $U$ into sets of measure $1 / n$. Set

$$
\Lambda_{0}(U)=\sum_{1}^{l} \Lambda_{0}\left(U_{j}\right)
$$

where $\Lambda_{0}\left(U_{j}\right), j=1, \ldots, l$, is defined as in Step 1 .

In view of Lemma $5.1(\mathrm{a}),(5.10)$ agrees with (5.2) in the case where $l / n=1 / q$ for some integer $q$.

For fixed $l$, the definition of $\Lambda_{0}(U)$, as given by (5.10), does not depend on the partition of $U$. Indeed, if $\left\{U_{1}^{\prime}, \ldots, U_{l}^{\prime}\right\}$ is another partition of $U$ into sets of measure $1 / n$ we have

$$
\sum_{1}^{l}\left[\Lambda_{0}\left(U_{j}\right)-\Lambda_{0}\left(U_{j}^{\prime}\right)\right]=\sum_{1}^{l} \Lambda\left(\chi_{U_{j}}-\chi_{U_{j}^{\prime}}\right)=0
$$

by Lemma 5.1(b) and Lemma 2.4 .

Finally if $l / n$ is replaced by $s l / s n$ (where $s$ is a positive integer), then Lemma 5.1(a) shows that the value of $\Lambda_{0}(U)$, given by (5.10), is unaffected.

LEMMA 5.2. (a) Let $U$ be a set $\tau$ such that $m(U)$ is rational and let $\left\{U^{1}, \ldots, U^{s}\right\}$ be a partition of $U$ into sets whose measure is rational. Then

$$
\Lambda_{0}(U)=\sum_{1}^{s} \Lambda_{0}\left(U^{\nu}\right)
$$


(b) If $U, V$ are sets in $\tau$ such that $m(U)=m(V)=$ rational number then

$$
\Lambda_{0}(U)-\Lambda_{0}(V)=\Lambda\left(\chi_{U}-\chi_{V}\right)
$$

Proof. (a) (5.11) is an immediate consequence of (5.10).

(b) Let $m(U)=m(V)=l / n$ where $l, n$ are positive integers. Let $\left\{U_{1}, \ldots\right.$, $\left.U_{l}\right\}$ and $\left\{V_{1}, \ldots, V_{l}\right\}$ be partitions of $U, V$ into sets of measure $1 / n$. Then, by Lemma 5.1(b) and Lemma 2.4,

$$
\Lambda_{0}(U)-\Lambda_{0}(V)=\sum_{1}^{l}\left[\Lambda_{0}\left(U_{i}\right)-\Lambda_{0}\left(V_{i}\right)\right]=\sum_{1}^{l} \Lambda\left(\chi_{U_{i}}-\chi_{V_{i}}\right)=\Lambda\left(\chi_{U}-\chi_{V}\right) .
$$

Up to this point we have used only properties (i) and (ii) of $\Lambda$. From this point on we shall use also the continuity of $\Lambda$, i.e. property (iii).

LEMMA 5.3. Let $\varepsilon>0$. If $U$ is a set in $\tau$ such that $m(U)$ is rational and $m(U) \leqslant \varepsilon$ then

$$
\left|\Lambda_{0}(U)\right| \leqslant \sup \left\{|\Lambda(h)|: h \in \varepsilon_{1}^{0}, m(K(h)) \leqslant 2 \varepsilon\right\} .
$$

Proof. Since $\Lambda_{0}(U)+\Lambda_{0}(I \backslash U)=\Lambda_{0}(I)=0$, it is sufficient to prove (5.13) in the case where $m(U) \leqslant \frac{1}{2}$.

Let $m(U)=l / n$ where $l, n$ are positive integers such that $l / n \leqslant \frac{1}{2}$. Let $\left\{U_{j}\right\}_{1}^{n}$ be a partition of $I$ into sets of equal measure such that $U_{1}^{l} U_{j}=U$. Then

$$
\Lambda_{0}(U)=\sum_{j=1}^{l} \Lambda_{0}\left(U_{j}\right)=\frac{1}{n} \sum_{j=1}^{l} \sum_{i=1}^{n} \Lambda\left(\chi_{U_{j}}-\chi_{U_{i}}\right)
$$

By Corollary 2.5 ,

$$
\sum_{j=1}^{l} \sum_{i=1}^{l} \Lambda\left(\chi_{U_{j}}-\chi_{U_{i}}\right)=0
$$

Hence,

$$
\Lambda_{0}(U)=\frac{1}{n} \sum_{j=1}^{l} \sum_{i=l+1}^{n} \Lambda\left(\chi_{U_{j}}-\chi_{U_{i}}\right)=\frac{1}{n} \sum_{s=1}^{n-l} \sum_{j=1}^{l} \Lambda\left(\chi_{U_{j}}-\chi_{U_{0,(j)+l}}\right),
$$

where $\sigma_{s}(j)$ is that integer between 1 and $n-l$ which equals $s+j \bmod (n$ $-l$ ). Hence, by Lemma 2.4,

$$
\Lambda_{0}(U)=\frac{1}{n} \sum_{s=1}^{n-l} \Lambda\left(\chi_{U}-\chi_{U_{s}^{*}}\right)
$$

where $U_{s}^{*} \subseteq I \backslash U$ for all $s$. Clearly (5.14) implies (5.13).

LEMMA 5.4. Let $U$ be a set in $\tau$ such that $m(U)$ is rational and let $\left\{U_{\nu}\right\}_{1}^{\infty}$ be a 
partition of $U$ consisting of sets of rational measure. Then

$$
\Lambda_{0}(U)=\sum_{1}^{\infty} \Lambda_{0}\left(U_{\nu}\right)
$$

Proof. Let $U^{s}=\cup_{\nu=1}^{s} U_{\nu}$. Then, by Lemma 5.2,

$$
\left\{\begin{array}{l}
\Lambda_{0}\left(U^{s}\right)=\sum_{1}^{s} \Lambda_{0}\left(U_{\nu}\right), \\
\Lambda_{0}(U)=\Lambda_{0}\left(U \backslash U^{s}\right)+\Lambda_{0}\left(U^{s}\right) .
\end{array}\right.
$$

By Lemma 5.3, $\lim _{s \rightarrow \infty} \Lambda_{0}\left(U \backslash U^{s}\right)=0$. Hence the required result.

STEP 3. Let $U$ be a set in $\tau$ and let $\left\{U_{\nu}\right\}_{1}^{\infty}$ be a partition of $U$ consisting of sets of rational measure. Set

$$
\Lambda_{0}(U)=\sum_{\nu=1}^{\infty} \Lambda_{0}\left(U_{\nu}\right)
$$

where $\Lambda_{0}\left(U_{\nu}\right), \nu=1,2, \ldots$, is defined as in Step 2.

We must show that this definition of $\Lambda_{0}(U)$ does not depend on the partition. But first we note that when $m(U)$ is rational, (5.16) agrees with the definition of $\Lambda_{0}(U)$ given in the previous step. This is clear in view of Lemma 5.4.

The following terminology will be useful. Given a set $U$ in $\tau$, a partition of $U$ consisting of sets of rational measure will be called rational partition of $U$. A set $E$ will be called an $r$-set if it is a finite union of intervals with rational end points.

LeMma 5.5. (a) Let $U$ be a set in $\tau$. The value $\Lambda_{0}(U)$ as given by (5.16) does not depend on the choice of the rational partition $\left\{U_{\nu}\right\}$.

(b) Let $U, V$ be sets in $\tau$ such that $m(U)=m(V)$. Then

$$
\Lambda_{0}(U)-\Lambda_{0}(V)=\Lambda\left(\chi_{U}-\chi_{V}\right)
$$

(c) $\Lambda_{0}$ is a signed measure on $\tau$.

(d) $\Lambda_{0}$ is absolutely continuous with respect to $m$. More precisely, given $\varepsilon>0$, if $U$ is in $\tau$ and $m(U) \leqslant \varepsilon$ then,

$$
\left|\Lambda_{0}(U)\right| \leqslant \sup \left\{\Lambda(h) \mid: h \in \mathcal{E}_{1}^{0}, m(K(h)) \leqslant 2 \varepsilon\right\} .
$$

Proof. (a) If $\left\{U_{\nu}\right\}_{1}^{\infty}$ is a disjoint sequence of sets of rational measure, then, in view of Lemmas 5.2 and 5.3 and Cauchy's criterion, the series $\Sigma_{1}^{\infty} \Lambda_{0}\left(U_{\nu}\right)$ converges. In fact it converges unconditionally.

Given $U$ in $\tau$, let $\left\{U_{\nu}\right\}_{1}^{\infty}$ and $\left\{U_{\nu}^{\prime}\right\}_{1}^{\infty}$ be two rational partitions of $U$ such that

$$
m\left(U_{\nu}\right)=m\left(U_{\nu}^{\prime}\right), \quad \nu=1,2, \ldots
$$


Then Lemma 5.2(b), Lemma 2.4 and the remark following it imply that

$$
\sum_{1}^{\infty} \Lambda_{0}\left(U_{v}\right)=\sum_{1}^{\infty} \Lambda_{0}\left(U_{\nu}^{\prime}\right)
$$

We now prove statement (a) in the case of an open set $\theta$ such that $m(\theta)$ is irrational. Given a rational partition $\left\{\theta_{\nu}\right\}_{1}^{\infty}$ of $\theta$ it can be seen that there exists a partition $\left\{\Theta_{\nu}^{\prime}\right\}_{1}^{\infty}$ of $\theta$ consisting of $r$-sets, such that $m\left(\mathcal{O}_{\nu}^{\prime}\right)=m\left(\mathcal{O}_{\nu}\right), \nu=1,2$, .... Therefore, in view of (5.20), it is sufficient to prove the following.

Let $\left\{\mathcal{O}_{v}^{\prime}\right\},\left\{\mathcal{O}_{v}^{\prime \prime}\right\}$ be two partitions of $\theta$ consisting of $r$-sets. Then

$$
\sum_{1}^{\infty} \Lambda_{0}\left(\mathcal{O}_{\nu}^{\prime}\right)=\sum_{1}^{\infty} \Lambda_{0}\left(\mathcal{O}_{\nu}^{\prime \prime}\right)
$$

Set $W_{\nu, \mu}=\mathcal{O}_{\nu}^{\prime} \cap \mathcal{O}_{\mu}^{\prime \prime}$. Then $\left\{W_{\nu, \mu}\right\}_{\nu, \mu=1}^{\infty}$ is a partition of $\mathcal{O}$ consisting of $r$-sets. The series $\sum_{\nu, \mu=1}^{\infty} \Lambda_{0}\left(W_{v, \mu}\right)$ converges unconditionally. Hence,

$$
\begin{aligned}
\sum_{\nu, \mu=1}^{\infty} \Lambda_{0}\left(W_{\nu, \mu}\right) & =\sum_{\nu=1}^{\infty} \sum_{\mu=1}^{\infty} \Lambda_{0}\left(W_{\nu, \mu}\right)=\sum_{1}^{\infty} \Lambda_{0}\left(\theta_{\nu}^{\prime}\right) \\
& =\sum_{\mu=1}^{\infty} \sum_{\nu=1}^{\infty} \Lambda_{0}\left(W_{\nu, \mu}\right)=\sum_{1}^{\infty} \Lambda_{0}\left(\left(_{\mu}^{\prime \prime}\right) .\right.
\end{aligned}
$$

Thus (a) is proved in the case that $U$ is open.

Now, consider an arbitrary set $U$ in $\tau$ and let $\theta$ be an open set such that $m(\theta)=m(U)$. Given a rational partition $\left\{U_{\nu}\right\}_{1}^{\infty}$ of $U$, let $\left\{\Theta_{\nu}\right\}$ be a rational partition of $\theta$ such that $m\left(U_{\nu}\right)=m\left(\mathcal{O}_{\nu}\right), \nu=1,2, \ldots$ Then, by Lemma 5.2(b), Lemma 2.4 and the remark following it, we have

$$
\sum_{1}^{\infty} \Lambda_{0}\left(U_{\nu}\right)-\sum_{1}^{\infty} \Lambda_{0}\left(\theta_{\nu}\right)=\sum_{1}^{\infty} \Lambda\left(\chi_{U_{\nu}}-\chi_{\theta_{\nu}}\right)=\Lambda\left(\chi_{U}-\chi_{\theta}\right) .
$$

By the previous part of the proof, the series $\Sigma_{1}^{\infty} \Lambda_{0}\left(\theta_{\nu}\right)$ depends on $\theta$ but not on the partition $\left\{\theta_{\nu}\right\}$. Therefore, $\Sigma_{1}^{\infty} \Lambda_{0}\left(U_{\nu}\right)$ is independent of the partition $\left\{U_{v}\right\}$ of $U$.

(b) Let $\left\{U_{\nu}\right\}_{1}^{\infty}$ and $\left\{V_{\nu}\right\}_{1}^{\infty}$ be rational partitions of $U, V$ such that $m\left(U_{\nu}\right)$ $=m\left(V_{\nu}\right), \nu=1,2, \ldots$. Then Lemma 5.2(b), Lemma 2.4 and the remark following it imply that

$$
\sum_{1}^{\infty} \Lambda_{p}\left(U_{\nu}\right)-\sum_{1}^{\infty} \Lambda_{0}\left(V_{\nu}\right)=\sum_{1}^{\infty} \Lambda\left(\chi_{U_{\nu}}-\chi_{V_{\nu}}\right)=\Lambda\left(\chi_{U}-\chi_{V}\right) .
$$

(c) By definition $\Lambda_{0}(\varnothing)=0$. The countable additivity of $\Lambda_{0}$ is easily verified using (5.16).

(d) The measure $\Lambda_{0}$ is finite (i.e., $\Lambda_{0}(U)$ is finite for every $U$ in $\tau$ ). Therefore $\Lambda_{0}$ is bounded. By definition (see (5.9)) $\Lambda_{0}(U)=0$ whenever $m(U)=0$. Thus 
$\Lambda_{0}$ is $m$-absolutely continuous. The inequality (5.18) was proved in the case where $m(U)$ is rational (Lemma 5.3). However, every set $U$ in $\tau$ is the limit of an increasing sequence of sets of rational measure. Therefore (5.18) holds for arbitrary sets in $\tau$.

The existence of a function $G$ with the properties stated in Theorem 4.4 follows (in the case $k=1$ ) from Lemma 5.5 and the Radon-Nikodým theorem. If $G_{1}, G_{2}$ are two such functions, then (setting $G^{*}=G_{1}-G_{2}$ ) we have,

$$
\int_{I} G^{*} f d m=0, \quad \forall f \in \mathcal{E}_{1}^{0} .
$$

But (5.23) implies that $G^{*}$ is a constant. Therefore the function $G$ in Theorem 4.4 (with $k=1$ ) is uniquely determined by (4.9) and the condition $\hat{\mu}_{1}(G)=0$.

This completes the proof of Theorem 4.4 in the case $k=1$.

6. Proof of Theorem 4.4 for $k>1$. As in the case $k=1$, the theorem is proved by constructing an appropriate signed measure $\Lambda_{0}$.

Let $E$ be a set in $\tau$ with $m(E)>0$ and let $\Sigma_{E}$ be a Blackwell $\sigma$-algebra for $E$ with respect to $\mu_{k}$. Note that

$$
\mu_{k}(U)=m(U)\left[\mu_{k}(E) / m(E)\right], \quad \forall U \in \Sigma_{E} .
$$

The measure space $\left(E, \Sigma_{E}, m\right)$ is separable, nonatomic and nonnegative. Therefore the measure algebra associated with this measure space is isomorphic with the measure algebra associated with the Lebesgue measure space of the interval $[0, m(E)]$, (see, e.g., $[6$, p. 173]). In view of this isomorphism the results proved in the previous section imply the following.

Given $\Lambda$ as in Theorem 4.4 and given a set $E$ and a $\sigma$-algebra $\Sigma_{E}$ as above, there exists a signed measure $\Lambda_{\Sigma_{E}}$ on $\left(E, \Sigma_{E}\right)$ such that:

$$
\begin{gathered}
\Lambda_{\Sigma_{E}}(E)=0 . \\
\Lambda_{\Sigma_{E}}(U)-\Lambda_{\Sigma_{E}}(V)=\Lambda\left(\chi_{U}-\chi_{V}\right) \\
\text { whenever } U, V \in \Sigma_{E} \text { and } \mu_{k}(U)=\mu_{k}(V) . \\
\Lambda_{\Sigma_{E}}(U)=0 \text { whenever } U \in \Sigma \text { and } m(U)=0 .
\end{gathered}
$$

Now let $\mathbb{Q}=\left\{A_{1}, \ldots, A_{k}\right\}$ be a $\mu_{k}$-independent family of sets such that $\sum_{1}^{k} m\left(A_{j}\right) \leqslant \frac{1}{2}$ and $\rho(\mathbb{Q}) \geqslant \rho_{k}\left(\frac{1}{2}\right)$ (see Lemma 3.1). Let $\Sigma_{j}$ be a Blackwell $\sigma$ algebra for $A_{j}$ and let $\Sigma$ be the direct sum of $\left\{\Sigma_{j}\right\}_{1}^{k}$. Let $\Lambda_{j}$ be a measure on $\left(A_{j}, \Sigma_{j}\right)$ satisfying (6.1)-(6.3) with $E$ and $\Sigma_{E}$ replaced by $A_{j}$ and $\Sigma_{j}$, $(j=1, \ldots, k)$. Finally let $\Lambda_{0}$ be the direct sum of the measures $\Lambda_{1}, \ldots, \Lambda_{k}$. Thus if $U \in \Sigma$ and $U=\cup_{1}^{k} U_{j}$ with $U_{j} \in \Sigma_{j}$, then 


$$
\Lambda_{0}(U)=\sum_{1}^{k} \Lambda_{j}\left(U_{j}\right)
$$

Our objective is to extend $\Lambda_{0}$ to all of $\tau$. The extension will be constructed in four steps.

STEP 1. Let $V$ be a set in $\tau$ such that $V \cap A_{0}=\varnothing$ (where $A_{0}=\cup_{1}^{k} A_{j}$ ). Assume that

$$
m(V) \leqslant \rho_{k}\left(\frac{1}{2}\right) \leqslant \rho(\mathbb{Q}) .
$$

Let $h=\chi_{S}-\chi_{T}$ be a $\Sigma$-representative of $V$. (By Corollary 3.3, such a representative exists.) Set

$$
\Lambda_{0}(V)=\Lambda\left(\chi_{V}-h\right)+\hat{\Lambda}_{0}(h)=\Lambda\left(\chi_{V}-h\right)+\Lambda_{0}(S)-\Lambda_{0}(T) .
$$

Since $S, T \in \Sigma, \Lambda_{0}(S)$ and $\Lambda_{0}(T)$ are defined by (6.4).

We shall show that the value of $\Lambda_{0}(V)$ as given by (6.6) does not depend on the choice of the $\Sigma$-representative $h$. Let $g$ be another $\Sigma$-representative of $V$. If $g, h$ are disjoint then

$$
\begin{aligned}
& \Lambda\left(\chi_{V}-h\right)+\hat{\Lambda}_{0}(h)-\Lambda\left(\chi_{V}-g\right)-\hat{\Lambda}_{0}(g) \\
& =\Lambda(g-h)+\hat{\Lambda}_{0}(h)-\hat{\Lambda}_{0}(g)=0,
\end{aligned}
$$

by Lemma 2.2 and (6.2). If $g, h$ are not disjoint we proceed as follows. Let $h=\chi_{E_{1}}-\chi_{E_{2}}, g=\chi_{F_{1}}-\chi_{F_{2}}$ where $E_{1} \cap E_{2}=F_{1} \cap F_{2}=\varnothing$. Denote $E_{0}$ $=I \backslash\left(E_{1} \cup E_{2}\right), F_{0}=I \backslash\left(F_{1} \cup F_{2}\right)$. Let $W_{i, j}=E_{i} \cap F_{j}$ and let $\left\{W_{i, j}^{\prime}, W_{i, j}^{\prime \prime}\right\}$ be a partition of $W_{i, j}$ into sets of equal $\mu_{k}$-measure belonging to $\Sigma$, $(i, j=0,1,2)$. Set $E_{i}^{\prime}=\bigcup_{j=0}^{2} W_{i, j}^{\prime}(i=1,2), F_{j}^{\prime}=\bigcup_{i=0}^{2} W_{i, j}^{\prime}(j=1,2)$ and $h^{\prime}=\chi_{E_{1}^{\prime}}-\chi_{E_{2}^{\prime}}, g^{\prime}=\chi_{F_{1}^{\prime}}-\chi_{F_{2}^{\prime}}$. Similarly we define $h^{\prime \prime}$ and $g^{\prime \prime}$. Finally, let $\left\{V^{\prime}, V^{\prime \prime}\right\}$ be a partition of $V$ into sets of equal $\mu_{k}$-measure. In view of the fact that $h^{\prime}, g^{\prime \prime}$ and $h^{\prime \prime}, g^{\prime}$ are disjoint pairs of functions we have

$$
\begin{aligned}
\Lambda\left(\chi_{V^{\prime}}-h^{\prime}\right)+\hat{\Lambda}_{0}\left(h^{\prime}\right) & =\Lambda\left(\chi_{V^{\prime}}-g^{\prime \prime}\right)+\hat{\Lambda}_{0}\left(g^{\prime \prime}\right), \\
\Lambda\left(\chi_{V^{\prime \prime}}-h^{\prime \prime}\right)+\hat{\Lambda}_{0}\left(h^{\prime \prime}\right) & =\Lambda\left(\chi_{V^{\prime \prime}}-g^{\prime}\right)+\hat{\Lambda}_{0}\left(g^{\prime}\right) .
\end{aligned}
$$

Summing up the two equalities we get

$$
\Lambda\left(\chi_{V}-h\right)+\hat{\Lambda}_{0}(h)=\Lambda\left(\chi_{V}-g\right)+\hat{\Lambda}_{0}(g) .
$$

Thus $\Lambda_{0}(V)$ is well defined by (6.6).

LEMMA 6.1. (a) If $U, V$ are two measurable sets contained in the complement of $A_{0}$, such that $\mu_{k}(U)=\mu_{k}(V)$ and (6.5) holds then

$$
\Lambda_{0}(U)-\Lambda_{0}(V)=\Lambda\left(\chi_{U}-\chi_{V}\right) .
$$


(b) If $V$ is a set as above and $\left\{V_{j}\right\}_{1}^{\infty}$ is a partition of $V$ then

$$
\Lambda_{0}(V)=\sum_{1}^{\infty} \Lambda_{0}\left(V_{j}\right)
$$

Proof. (a) Let $h$ be a $\Sigma$-representative of $U$ and $V$. Then

$$
\begin{aligned}
\Lambda_{0}(U)-\Lambda_{0}(V) & =\Lambda\left(\chi_{U}-h\right)+\hat{\Lambda}_{0}(h)-\hat{\Lambda}_{0}\left(\chi_{V}-h\right)-\hat{\Lambda}_{0}(h) \\
& =\Lambda\left(\chi_{U}-\chi_{V}\right) .
\end{aligned}
$$

(b) Let $\left\{h_{j}\right\}$ be a family of disjoint functions such that $h_{j}$ is a $\Sigma$-representative of $V_{j}$. Such a family of functions exists by Corollary 3.3. Then (setting $\left.h=\Sigma_{1}^{\infty} h_{j}\right)$ we have

$$
\Lambda_{0}(V)=\Lambda\left(\chi_{V}-h\right)+\hat{\Lambda}_{0}(h)=\sum_{1}^{\infty} \Lambda\left(\chi_{V_{j}}-h_{j}\right)+\sum_{1}^{\infty} \hat{\Lambda}_{0}\left(h_{j}\right)=\sum_{1}^{\infty} \Lambda_{0}\left(V_{j}\right) .
$$

Here we used the countable disjoint additivity of $\Lambda$ (properties (i) and (iii)) and the fact that $\Lambda_{0}$ is a measure on $\Sigma$.

STEP 2. Let $V$ be a measurable set contained in the complement of $A_{0}$. Let $r$ be a positive integer such that $1 / r \leqslant \rho_{k}\left(\frac{1}{2}\right)$. Let $\left\{V_{1}, \ldots, V_{r}\right\}$ be a partition of $V$ into sets of equal $\mu_{k}$-measure. Then each of the sets $V_{i}$ satisfies (6.5) so that $\Lambda_{0}\left(V_{i}\right)(i=1, \ldots, r)$ is defined by (6.6). Set

$$
\Lambda_{0}(V)=\sum_{1}^{r} \Lambda_{0}\left(V_{i}\right)
$$

For a given $r$, the value of $\Lambda_{0}(V)$ as given by (6.9) does not depend on the partition. This follows from Lemma 2.4 and Lemma 6.1(a). Further, using Lemma 6.1(b) it is easy to verify that the above definition of $\Lambda_{0}(V)$ does not depend on $r$. Finally, if $V$ satisfies (6.5) then $\Lambda_{0}(V)$ as given by (6.9) coincides with $\Lambda_{0}(V)$ as defined in the previous step. This is clear in view of Lemma 6.1(b).

Lemma 6.2. The statements of Lemma 6.1 are valid even if $U, V$ do not satisfy condition (6.5).

Proof. This is an immediate consequence of (6.9), Lemmas 6.1 and 2.4.

STEP 3. Let $V$ be a set in $\tau$ such that

$$
m(V) \leqslant \rho_{k}(1 / 4) .
$$

Since $\rho_{k}(1 / 4) \leqslant 1 / 4$, we have $m\left(V \cup A_{0}\right) \leqslant 3 / 4$. Therefore, by Lemma 3.1 and Corollary 3.3, there exists a function $h$ of the form $\chi_{S}-\chi_{T}(S \cap T=\varnothing)$ such that

$$
\hat{\mu}_{k}(h)=\mu_{k}(V) \text { and } K(h) \cap\left(V \cup A_{0}\right)=\varnothing .
$$


Set

$$
\Lambda_{0}(V)=\Lambda\left(\chi_{V}-h\right)+\hat{\Lambda}_{0}(h),
$$

where $\hat{\Lambda}_{0}(h)=\Lambda_{0}(S)-\Lambda_{0}(T)$ is defined as in Step 2.

If $V \cap A_{0}=\varnothing$ then, in view of Lemma 6.2, this definition of $\Lambda_{0}(V)$ agrees with that given in Step 2.

The value of $\Lambda_{0}(V)$ given by (6.12) does not depend on the choice of $h$. This is proved by the same argument that was used in Step 1 to show that $\Lambda_{0}(V)$ is well defined.

LEMma 6.3. (a) If $U, V$ are sets in $\tau$ of equal $\mu_{k}$-measure, such that

$$
m(U) \leqslant \rho_{k}(1 / 8) \text { and } m(V) \leqslant \rho_{k}(1 / 8)
$$

then

$$
\Lambda_{0}(U)-\Lambda_{0}(V)=\Lambda\left(\chi_{U}-\chi_{V}\right)
$$

(b) If $V$ is a set in $\tau$ satisfying (6.10) and if $\left\{V_{j}\right\}_{1}^{\infty}$ is a partition of $V$ then

$$
\Lambda_{0}(U)=\sum_{1}^{\infty} \Lambda_{0}\left(v_{j}\right)
$$

Proof. (a) Since $\rho_{k}(1 / 8) \leqslant 1 / 8, m(U \cup V) \leqslant 1 / 4$. Therefore there exists a function $h$ of the form $\chi_{S}-\chi_{T}, S \cap T=\varnothing$, such that $h$ satisfies (6.11) with respect to $V$ and $U$. Thus

$$
\Lambda_{0}(U)-\Lambda_{0}(V)=\Lambda\left(\chi_{U}-h\right)+\hat{\Lambda}_{0}(h)-\Lambda\left(\chi_{V}-h\right)-\hat{\Lambda}_{0}(h)=\Lambda\left(\chi_{U}-\chi_{V}\right) .
$$

(b) This statement can be proved in essentially the same way as Lemma 6.1(b).

STEP 4. Let $V$ be a set in $\tau$. Let $s$ be a positive integer such that $1 / s \leqslant \rho_{k}(1 / 8)$ and let $\left\{V_{1}, \ldots, V_{s}\right\}$ be a partition of $V$ into sets of equal $\mu_{k}$ measure. Set

$$
\Lambda_{0}(V)=\sum_{1}^{s} \Lambda_{0}\left(V_{i}\right),
$$

where $\Lambda_{0}\left(V_{i}\right)$ is defined as in Step 3.

For a given $s$, the value of $\Lambda_{0}(V)$ does not depend on the partition. This follows from Lemma 2.4 and Lemma 6.3(a). Further, the value of $\Lambda_{0}(V)$ does not depend on $\Sigma$. This is easily verified, using Lemma 6.3(b). Finally, if $V$ satisfies condition (6.10) then $\Lambda_{0}(V)$ as given by (6.16) coincides with the value of $\Lambda_{0}(V)$ as defined in Step 3. This is clear in view of Lemma 6.3(b).

LEMMA 6.4. (a) If $U, V$ are sets in $\tau$ of equal $\mu_{k}$-measure then (6.14) holds. 
(b) If $V$ is a set in $\tau$ and $\left\{V_{j}\right\}_{1}^{\infty}$ a partition of $V$ then (6.15) holds.

Proof. This is an immediate consequence of (6.16), Lemmas 6.3 and 2.4.

We can now complete the proof of Theorem 4.4. In view of (6.3) and the construction of $\Lambda_{0}$ we have

$$
\Lambda_{0}(U)=0 \text { whenever } U \text { is an } m \text {-null set. }
$$

This together with Lemma 6.4 implies that $\Lambda_{0}$ is an $m$-absolutely continuous signed measure on $(I, \tau)$. Therefore, by the Radon-Nikodým theorem there exists a function $G$ in $L^{1}(I)$ such that

$$
\Lambda_{0}(U)=\int_{U} G d m, \quad \forall U \in \tau .
$$

Lemma 6.4(a) and (6.18) imply that $G$ satisfies (4.9). Clearly if $q$ is a polynomial of order less than $k$ then

$$
\int_{I} q f d m=0, \quad \forall f \in \mathcal{E}_{k}^{0} .
$$

Let $q$ be the (unique) polynomial of order less than $k$ such that $\hat{\mu}_{k}(G)$ $=\hat{\mu}_{k}(q)$. Then the function $G^{*}=G-q$ satisfies (4.9) and the condition $\hat{\mu}_{k}\left(G^{*}\right)=0$. Finally, the uniqueness of $G^{*}$ is an immediate consequence of the following

LEMMA 6.5. Let $g$ be in $L^{1}(I)$ and suppose that

$$
\int_{I} g f d m=0, \quad \forall f \in \mathcal{E}_{k}^{0} .
$$

Then $g$ is a polynomial of order less than $k$.

A proof of this lemma will be given in the Appendix.

7. A representation theorem for $\mu_{k}$-invariant functionals. With the proof of Theorem 4.4 we have completed the first part of our program for the proof of Theorem 4.1. One of the main features in the second part of our program, as described in $\$ 4$, is a representation theorem for $\mu_{k}$-invariant functionals. The present section is devoted to the development of this result.

THEOREM 7.1. Let $\hat{N}$ be a functional on $\delta_{k}$ possessing the following properties:

(a) $\hat{N}$ is disjointly additive.

(b) $\hat{N}$ is continuous with respect to the (bm) topology.

(c) $\hat{N}$ is $\mu_{k}$-invariant.

Then there exists a function $\hat{H}: I \times R \rightarrow R$ and functions $\eta_{j}: R \rightarrow R$ such that 


$$
\left\{\begin{array}{l}
\hat{H(t, 0)}=0, \\
\hat{H}(t, a)=\sum_{1}^{k} \eta_{j}(a) t^{j-1}, \quad \forall a \in R \text { and } \forall t \in I,
\end{array}\right.
$$

and

$$
\hat{N}(f)=\int_{I} \hat{\mathbf{H}}(f) d m, \quad \forall f \in \delta_{k} .
$$

Remark. Assumption (b) may be replaced by a weaker continuity condition, as follows.

Given two positive numbers $a, b$, denote

$$
\begin{aligned}
& \delta(\varepsilon: a, b)=\sup \left\{|\hat{N}(f)|: f \in \mathcal{S}_{k}, \text { range }(f) \subseteq\{0, a,-b\},\right. \\
& m(K(f)) \leqslant \varepsilon\} .
\end{aligned}
$$

Then (b) may be replaced by

(b') $\lim _{\varepsilon \rightarrow 0} \delta(\varepsilon ; a, b)=0$, for every pair of positive numbers $a, b$.

Proof. Let $a, b$, be positive numbers. Given a set $S$ in $\tau$, let $\left\{S_{1}, S_{2}\right\}$ be a partition of $S$ such that

$$
\mu_{k}\left(S_{1}\right)=[b /(a+b)] \mu_{k}(S) .
$$

Such a partition will be called an $(a, b)$ partition. By property (c), $\hat{N}\left(a \chi_{S_{1}}-b \chi_{S_{2}}\right)$ is independent of the particular choice of the $(a, b)$ partition. In fact this expression depends only on $\mu_{k}(S)$.

Let

$$
\chi_{a, b}(S)=(a+b) \hat{N}\left(a \chi_{S_{1}}-b \chi_{S_{2}}\right) .
$$

Then $\gamma_{a, b}$ is a set function on $\tau$. Properties (a) and ( $\left.b^{\prime}\right)$ imply that $\gamma_{a, b}$ is countably additive and absolutely continuous with respect to Lebesgue measure. Therefore, by the Radon-Nikodým theorem, there exists an element $F_{a, b}$ of $L^{1}(I)$ such that

$$
\gamma_{a, b}(S)=\int_{S} F_{a, b} d m, \quad \forall S \in \tau .
$$

Since

$$
\chi_{a, b}(U)=\chi_{a, b}(V) \text { whenever } U, V \in \tau \text { and } \mu_{k}(U)=\mu_{k}(V),
$$

it follows that

$$
\int_{I} F_{a, b} h d m=0, \quad \forall h \in \mathcal{E}_{k}^{0} .
$$


Thus, by Lemma $6.5, F_{a, b}$ is a polynomial of order less than $k$. Set

$$
F_{a, b}(t)=\sum_{1}^{k} \xi_{j}(a, b) t^{j-1}, \quad \forall t \in I .
$$

In order to obtain from (7.7) a kernel satisfying (7.1) we have to show, in effect, that the coefficients $\xi_{j}$ can be expressed in terms of functions $\eta_{j}$ of one variable.

We shall consider separately the case where $\hat{N}$ is even and the case where $\hat{N}$ is odd. It is clear that the validity of the theorem in these two particular cases implies its validity in the general case.

First, assume that $\hat{N}$ is even. Note that the set function $\gamma_{a, a} / 2 a(a>0)$ defined above then coincides with the set function $\gamma_{a}$ defined in Lemma 2.1 (see (2.8)). Therefore, if $f=\sum_{i=1}^{n} \alpha_{i} \chi_{E_{i}} \in \mathcal{S}_{k}$, Lemma 2.1 and (7.5) imply that

$$
\hat{N}(f)=\sum_{i=1}^{n} \gamma_{\left|\alpha_{i}\right|}\left(E_{i}\right)=\sum_{i=1}^{n} \frac{1}{2\left|\alpha_{i}\right|} \int_{E_{i}} F_{\left|\alpha_{i}\right|,\left|\alpha_{i}\right|} d m .
$$

Hence, setting

$$
\left\{\begin{array}{l}
\hat{H}(\cdot, a)=\frac{1}{2|a|} F_{|a|,|a|}, \quad a \neq 0, \\
\hat{H}(\cdot, 0)=0,
\end{array}\right.
$$

we get (7.2). Furthermore, by (7.7) the function $\hat{H}$ defined above satisfies condition (7.1).

Next suppose that $\hat{N}$ is odd. We claim that, in this case, the equality

$$
a \bar{\xi}(b, c)+c \bar{\xi}(a, b)+b \bar{\xi}(c, a)=0,
$$

holds for every triple of positive numbers $a, b, c$, where $\bar{\xi}=\left(\xi_{1}, \ldots, \xi_{k}\right)$. Since the equality is symmetric in its variables, we may and shall assume that $c \geqslant \max (a, b)$.

Given a set $S$ let $\left\{S_{1}, S_{2}\right\}$ be a partition of $S$ into sets of equal $\mu_{k}$-measure. Further, let $\left\{S_{1}^{\prime}, S_{1}^{\prime \prime}\right\}$ be an $(a, b)$ partition of $S_{1}$ and let $S_{2}^{\prime}$ be a subset of $S_{2}$ such that $\mu_{k}\left(S_{2}^{\prime}\right)=[a b / c(a+b)] \mu_{k}\left(S_{2}\right)$. Then, by (7.4),

$$
\begin{aligned}
\chi_{a, b}\left(S_{1}\right) & =(a+b) \hat{N}\left(a \chi_{S_{1}^{\prime}}-b \chi_{S_{1}^{\prime \prime}}\right), \\
\gamma_{c, a}\left(S_{1}^{\prime} \cup S_{2}^{\prime}\right) & =(a+c) \hat{N}\left(c \chi_{S_{2}^{\prime}}-a \chi_{S_{1}^{\prime}}\right), \\
\gamma_{b, c}\left(S_{1}^{\prime \prime} \cup S_{2}^{\prime}\right) & =(b+c) \hat{N}\left(b \chi_{S_{1}^{\prime \prime}}-c \chi_{S_{2}^{\prime}}\right) .
\end{aligned}
$$

However by (7.5) and (7.7)

$$
\chi_{a, b}(U)=\bar{\xi}(a, b) \cdot \mu_{k}(U), \quad \forall U \in \tau .
$$


Therefore, setting $x=\mu_{k}(S)$, we obtain

where

$$
\begin{aligned}
\bar{\xi}(a, b) \cdot(x / 2) & =(a+b) \hat{N}\left(a \chi_{S_{1}^{\prime}}-b \chi_{S_{1}^{\prime \prime}}\right), \\
\bar{\xi}(c, a) \cdot(\beta x / 2) & =(a+c) \hat{N}\left(c \chi_{S_{2}^{\prime}}-a \chi_{S_{1}^{\prime}}\right), \\
\bar{\xi}(b, c) \cdot\left(\beta^{\prime} x / 2\right) & =(b+c) \hat{N}\left(b \chi_{S_{1}^{\prime \prime}}-c \chi_{S_{2}^{\prime}}\right),
\end{aligned}
$$

$$
\beta=\frac{(a+c) b}{(a+b) c} \text { and } \beta^{\prime}=\frac{(b+c) a}{(a+b) c} \text {. }
$$

Thus,

$$
\begin{aligned}
& {[c \bar{\xi}(a, b)+b \bar{\xi}(c, a)+a \bar{\xi}(b, c)] \cdot x} \\
& \quad=2(a+b) c\left[\hat{N}\left(a \chi_{S_{1}^{\prime}}-b \chi_{S_{1}^{\prime \prime}}\right)+\hat{N}\left(c \chi_{S_{2}^{\prime}}-a \chi_{S_{1}^{\prime}}\right)+\hat{N}\left(b \chi_{S_{1}^{\prime \prime}}-c \chi_{S_{2}^{\prime}}\right)\right] .
\end{aligned}
$$

Since $\hat{N}$ is odd, it follows from Lemma 2.2 that the right-hand side of (7.9) is zero. Note also that (7.9) holds for every $x$ in the range of $\mu_{k}$, which is a $k$ dimensional subset of $R_{k}$. These facts imply (7.8).

Let $c_{0}$ be a fixed positive number and set

$$
\bar{\eta}(a)=-\bar{\eta}(-a)=\frac{1}{c_{0}} \bar{\xi}\left(a, c_{0}\right), \quad \forall a>0 \text {, and } \bar{\eta}(0)=\dot{0} .
$$

The fact that $\hat{N}$ is odd implies that $\gamma_{a, b}=-\gamma_{b, a}$, and hence $\bar{\xi}(a, b)=-\bar{\xi}(b, a)$, $(a, b>0)$. Therefore, setting $c=c_{0}$ in (7.8) and solving for $\bar{\xi}(a, b)$, we obtain

$$
\bar{\xi}(a, b)=b \bar{\eta}(a)-a \bar{\eta}(b), \quad \forall a, b>0 .
$$

Let $\hat{H}$ be the function given by

$$
\hat{H}(t, a)=\sum_{1}^{k} \eta_{j}(a) t^{j-1}, \quad \forall a \in R, \forall t \in I,
$$

where $\bar{\eta}(a)=\left(\eta_{1}(a), \ldots, \eta_{k}(a)\right)$. Then, by (7.4), (7.5), (7.7) and (7.11), we deduce that

$$
\begin{aligned}
\hat{N}\left(a \chi_{U}-b \chi_{V}\right) & =\frac{1}{a+b} \int_{U \cup V} F_{a, b} d m=\frac{1}{a+b} \bar{\xi}(a, b) \cdot \mu_{k}(U \cup V) \\
& =\bar{\eta}(a) \cdot \mu_{k}(U)-\bar{\eta}(b) \cdot \mu_{k}(V),
\end{aligned}
$$

whenever $a, b$ are positive and $U, V$ are disjoint sets such that $a \chi_{U}-b \chi_{V}$ $\in \delta_{k}$. Now (7.10), (7.12) and (7.13) imply that

$$
\hat{N}(f)=\int_{I} \hat{\mathbf{H}}(f) d m, \quad \forall f \in \mathcal{E}_{k} .
$$


Finally, since $\hat{H}$ satisfies (7.1) and (7.14), Lemma 4.3 implies that $\hat{H}$ satisfies (7.2). This completes the proof of the theorem.

8. Proof of Theorem 4.1. Let $N$ be a functional satisfying the assumptions of the theorem. Let $N_{\mathrm{e}}$ and $N_{\mathrm{o}}$ be the even and odd components of $N$, respectively, i.e.

$$
\left\{\begin{array}{l}
N_{\mathrm{e}}(f)=[N(f)+N(-f)] / 2, \\
N_{\mathrm{o}}(f)=[N(f)-N(-f)]^{\prime} 2, \quad \forall f \in \aleph_{k} .
\end{array}\right.
$$

It is clear that both $N_{\mathrm{e}}$ and $N_{\mathrm{o}}$ satisfy assumptions (i) and (ii). By Lemma 4.2 there exists a kernel for $N_{\mathrm{e}}$. We proceed to prove the existence of a kernel for $N_{\mathrm{o}}$. Obviously, the sum of the two kernels will be a kernel for $N$.

Given a real number $a$ we define a functional $\Lambda_{a}$ on $\varepsilon_{k}^{0}$ by

$$
\Lambda_{a}(f)=N_{\mathrm{o}}(a f), \quad \forall f \in \mathcal{E}_{k}^{0} .
$$

This functional satisfies the assumptions of Theorem 4.4. Therefore there exists a unique element $G(\cdot, a)$ in $L^{1}(I)$ such that $\hat{\mu}_{k}(G(\cdot, a))=0$ and

$$
\Lambda_{a}(f)=\int_{l} \mathbf{G}(f) d m, \quad \forall f \in \varepsilon_{k} .
$$

Since $\Lambda_{a}=-\Lambda_{-a}$ we have

$$
G(\cdot, a)=-G(\cdot,-a), \quad \forall a \in R .
$$

Now we define a functional $\hat{N}$ on $\varsigma_{k}$ as follows:

$$
\hat{N}(h)=N_{\mathrm{o}}(h)-\int_{l} \mathbf{G}(h) d m, \quad \forall h \in \varsigma_{k} .
$$

We claim that $\hat{N}$ is odd and that it satisfies conditions (a), (b'), (c) of Theorem 7.1 and the remark following it. The fact that $\hat{N}$ is odd and disjointly additive is clear from its definition, together with (8.1) and (8.4). The continuity of $N$ and the fact that $G(\cdot, a) \in L^{1}(I)$ for every real $a$, imply that $\hat{N}$ satisfies ( $\left.\mathrm{b}^{\prime}\right)$. We proceed to show that $\hat{N}$ satisfies condition (c).

First we note that, by $(8.2)-(8.5), \hat{N}(f)=0$ for every function $f$ which is a linear combination of disjoint functions in $\mathcal{E}_{k}^{0}$. Now suppose that $f$ and $g$ are two functions in $\varsigma_{k}$ such that

$$
\mu_{k}\left(f^{-1}(a)\right)=\mu_{k}\left(g^{-1}(a)\right), \quad \forall a \in R .
$$

If $f$ and $g$ are disjoint, then $f-g$ is a linear combination of disjoint functions in $\varepsilon_{k}^{0}$, so that

$$
\hat{N}(f)-\hat{N}(g)=\hat{N}(f-g)=0 .
$$


Next we consider the general case, when $f$ and $g$ are not necessarily disjoint. Let

$$
\begin{cases}\operatorname{range}(f)=\operatorname{range}(g)=\left\{a_{1}, \ldots,\right. & \left.a_{n}\right\}, \\ f^{-1}\left(a_{i}\right)=S_{i}, g^{-1}\left(a_{i}\right)=T_{i}, & (i=1, \ldots, n) \\ W_{i, j}=S_{i} \cap T_{j}, & (i, j=1, \ldots, n) .\end{cases}
$$

Let $\left\{W_{i, j}^{1}, W_{i, j}^{2}\right\}$ be a partition of $W_{i, j}$ into sets of equal $\mu_{k}$-measure and set

$$
S_{i}^{l}=\bigcup_{j=1}^{n} W_{i, j}^{l}, \quad f_{l}=\sum_{1}^{n} a_{i} \chi_{S_{i}^{\prime}}
$$

and

$$
T_{j}^{l}=\bigcup_{i=1}^{n} W_{i, j}^{l}, \quad g_{l}=\sum_{i}^{n} a_{j} \chi_{T_{j}}, \quad(l=1,2) .
$$

Then each of the pairs $f_{1}, g_{2}$ and $f_{2}, g_{1}$ consists of disjoint functions which satisfy (8.6). Hence, by (8.7),

$$
\hat{N}\left(f_{1}\right)=\hat{N}\left(g_{2}\right) ; \hat{N}\left(f_{2}\right)=\hat{N}\left(g_{1}\right) .
$$

Summing up these two equalities and using disjoint additivity we obtain

$$
\hat{N}(f)=\hat{N}(g) .
$$

Thus $\hat{N}$ satisfies condition (c).

By Theorem 7.1 and the remark following it, there exists a kernel $\hat{H}$ for $\hat{N}$. By (8.5), the function $H_{\mathrm{o}}=\hat{H}+G$ is a kernel for $N_{\mathrm{o}}$. This completes the proof of the existence of a kernel for $N$.

If $q$ is a polynomial of order less than $k$ and $H_{q}$ is defined as in (1.1) then, clearly,

$$
\int_{I} \mathbf{H}_{q}(f) d m=0, \quad \forall f \in \varsigma_{k} .
$$

We shall show that, conversely, if $H$ is a real function on $I \times R$ satisfying (4.1) and

$$
\int_{I} \mathbf{H}(f) d m=0, \quad \forall f \in \mathfrak{s}_{k}
$$

then $H$ is equivalent to $H_{q}$ for some polynomial $q$ of order less than $k$.

In order to prove this statement we consider separately the odd and even parts of $H$, defined as follows, 


$$
\left\{\begin{array}{l}
H_{\mathrm{e}}(\cdot, a)=[H(\cdot, a)+H(\cdot,-a)] / 2 \\
H_{\mathrm{o}}(\cdot, a)=[H(\cdot, a)-H(\cdot,-a)] / 2 .
\end{array}\right.
$$

Let $U \in \tau$ and let $\left\{U_{1}, U_{2}\right\}$ be a partition of $U$ into sets of equal $\mu_{k}$-measure. Then (8.9) and (8.10) imply that

$$
\int_{U} H_{\mathrm{e}}(t, a) d m=\int_{U_{1}} H_{\mathrm{e}}(t, a) d m+\int_{U_{2}} H_{\mathrm{e}}(t,-a) d m=0, \quad \forall a \in R .
$$

Thus, for every real $a$,

$$
H_{\mathrm{e}}(t, a)=0 \text { for a.e. } t \text { in } I .
$$

By (8.9) and (8.10),

$$
\int_{I} H_{\mathrm{o}}(\cdot, a) f d m=\int_{I} \mathbf{H}_{\mathrm{o}}(a f) d m=0, \quad \forall f \in \mathcal{E}_{k}^{0}, \forall a \in R .
$$

Hence, by Lemma $6.5, H_{0}(\cdot, a)$ is equivalent to a polynomial of order less than $k$, for every real $a$. Let

$$
H_{0}(t, a)=\sum_{1}^{k} c_{j}(a) t^{j-1}, \text { for a.e. } t \text { in } I, \text { for } \forall a \in R .
$$

Set $\bar{c}(a)=\left(c_{1}(a), \ldots, c_{k}(a)\right)$ and note that $-\bar{c}(a)=\bar{c}(-a)$.

Let $a, b$ be two positive numbers. Let $U$ be in $\tau$ and let $\left\{U_{1}, U_{2}\right\}$ be an $(a, b)$ partition of $U$, with respect to $\mu_{k}$. (The definition of an $(a, b)$ partition is given in the proof of Theorem 7.1.) Then by (8.9), (8.10) and (8.12) we have

$$
\bar{c}(a) \cdot \mu_{k}\left(U_{1}\right)-\bar{c}(b) \cdot \mu_{k}\left(U_{2}\right)=0 .
$$

Hence, $[b \bar{c}(a)-a \bar{c}(b)] \cdot \mu_{k}(U)=0$. Since the range of $\mu_{k}$ is $k$-dimensional, it follows that

$$
b \bar{c}(a)-a \bar{c}(b)=0, \quad \forall a, b>0 .
$$

This, together with the fact that $\bar{c}$ is odd, implies that

$$
\bar{c}(a)=a \bar{C},
$$

for some fixed vector $\bar{C}=\left(c_{1}, \ldots, c_{k}\right)$. Let $q(t)=\Sigma_{1}^{k} c_{j} t^{j-1}, t \in I$. Then (8.12) and (8.13) imply that $H_{\mathrm{o}}$ and $H_{q}$ are equivalent. Therefore, in view of (8.11), $H$ and $H_{q}$ are equivalent. This completes the proof of the theorem.

9. Continuity properties of the kernel; completion of proof of Theorem 1.3. In the previous sections we established the existence of a kernel (in the sense of Theorem 4.1) for continuous, disjointly additive functionals on $\delta_{k}$. In this section we obtain certain continuity properties of the kernel or, more precisely, 
of an operator defined by it. Then, using these properties, we show that, under the assumptions of Theorem 1.3, this kernel is equivalent to a Carathéodory kernel.

LEMMA 9.1. Let $N$ be a functional on $\S_{k}$ satisfying the assumptions of Theorem 4.1 and let $H$ be a kernel for $N$.

(a) If $\left\{f_{n}\right\}$ is a (bm)-convergent sequence of simple functions, then $\left\{\mathbf{H}\left(f_{n}\right)\right\}$ is a Cauchy sequence in $L^{1}(I)$.

(b) Suppose that, in addition to the previous assumptions, $N$ is continuous on $\delta_{k}$ with respect to the $L^{p}$ norm, $p \in[1, \infty)$. In this case, if $\left\{f_{n}\right\}$ is an $L^{p}$-convergent sequence of simple functions, then $\left\{\mathbf{H}\left(f_{n}\right)\right\}$ is a Cauchy sequence in $L^{1}(I)$.

(c) In both cases, if the sequence converges to a simple function $f$, then $L^{1}-\lim \mathbf{H}\left(f_{n}\right)=\mathbf{H}(f)$.

Proof. We shall prove (a) and (b). Statement (c) is an immediate consequence of the previous two. The argument given below will refer simultaneously to both (a) and (b), unless otherwise stated.

Set $D=\cup_{1}^{\infty} K\left(f_{n}\right)$. We note that, in view of the fact that $H(\cdot, 0)=0$, it is sufficient to prove (a) and (b) in the case where $m(D) \leqslant \frac{1}{2}$. Therefore we assume that this holds.

Let $f=\lim f_{n}$ (with respect to $(\mathrm{bm})$-convergence in (a) and $L^{p}$-convergence in (b)). Obviously $K(f) \subseteq D$. Let $E$ be a set in $\tau$, disjoint from $D$, such that $m(E)=\frac{1}{4}$. By Lemmas 3.1 and 3.2, if $M$ is a sufficiently large positive number, there exists a measurable simple function $h$ such that

$$
h=M\left(\chi_{U}-\chi_{V}\right), \quad K(h) \subseteq E, \quad \hat{\mu}_{k}(h)=\hat{\mu}_{k}(f) .
$$

Set $f_{n}^{*}=f_{n}-h, f^{*}=f-h$. Note that $\left\{f_{n}^{*}\right\}$ also satisfies the conditions of (a) (respectively (b)) and

$$
\begin{cases}\lim f_{n}^{*}=f^{*} & \text { (with respect to }(b m) \text {-convergence } \\ & \text { in (a) and } L^{p} \text {-convergence in (b)), } \\ f^{*} \in \Re_{k}^{p} & (\text { with } p=\infty \text { in }(\text { a) }), \\ m\left(D^{*}\right) \leqslant \frac{3}{4}, & \text { where } D^{*}=\bigcup_{1}^{\infty} K\left(f_{n}^{*}\right) .\end{cases}
$$

We proceed to prove that $\left\{\mathbf{H}\left(f_{n}^{*}\right)\right\}$ is a Cauchy sequence in $L^{1}(I)$. Clearly, this will imply that $\left\{\mathbf{H}\left(f_{n}\right)\right\}$ is a Cauchy sequence in $L^{\mathrm{I}}(I)$.

Denote

$$
\begin{aligned}
& E_{i, j}^{+}=\left\{t \in[0,1]: \mathbf{H}\left(f_{i}^{*}\right)(t)-\mathbf{H}\left(f_{j}^{*}\right)(t) \geqslant 0\right\}, \\
& E_{i, j}^{-}=[0,1] \backslash E_{i, j}^{+} .
\end{aligned}
$$

Then 


$$
I_{i, j}=\int_{I}\left|\mathbf{H}\left(f_{i}^{*}\right)-\mathbf{H}\left(f_{j}^{*}\right)\right| d m=\int_{I}\left[\mathbf{H}\left(g_{i, j}\right)-\mathbf{H}\left(h_{i, j}\right)\right] d m
$$

where

$$
g_{i, j}=f_{i}^{*} \chi_{E_{i, j}^{+}}+f_{j}^{*} \chi_{E_{i, j}} \text { and } h_{i, j}=f_{j}^{*} \chi_{E_{i, j}^{+}}+f_{i}^{*} \chi_{E_{l, j}} .
$$

Clearly, (9.2) implies that

$$
\lim _{i, j \rightarrow \infty} g_{i, j}=\lim _{i, j \rightarrow \infty} h_{i, j}=f^{*},
$$

with respect to $(b m)$-convergence in (a) and $L^{p}$-convergence in (b).

In addition, in case $(\mathrm{a}),\left\{g_{i, j}\right\}$ and $\left\{h_{i, j}\right\}$ are bounded families of functions. This together with (9.2) and (9.5) implies that

$$
\lim _{i, j \rightarrow \infty} \hat{\mu}_{k}\left(g_{i, j}\right)=\lim _{i, j \rightarrow \infty} \hat{\mu}_{k}\left(h_{i, j}\right)=\hat{\mu}_{k}\left(f^{*}\right)=0 .
$$

Hence, in view of the fact that $m\left(D^{*}\right)<1$, if $i, j$ are sufficiently large, (say $i, j \geqslant n_{0}$ ), there exist simple measurable functions $\bar{g}_{i, j}$ and $\bar{h}_{i, j}$ such that

$$
\left\{\begin{array}{l}
\bar{g}_{i, j} \text { and } \bar{h}_{i, j} \text { are each a difference of two characteristic functions, } \\
K\left(\bar{g}_{i, j}\right) \cap D^{*}=K\left(\bar{h}_{i, j}\right) \cap D^{*}=\varnothing \\
\lim _{i, j \rightarrow \infty} m\left(K\left(\bar{g}_{i, j}\right)\right)=\lim _{i, j \rightarrow \infty} m\left(K\left(\bar{h}_{i, j}\right)\right)=0 \\
\hat{\mu}_{k}\left(g_{i, j}\right)=\hat{\mu}_{k}\left(\bar{g}_{i, j}\right), \quad \hat{\mu}_{k}\left(h_{i, j}\right)=\hat{\mu}_{k}\left(\bar{h}_{i, j}\right)
\end{array}\right.
$$

This is a consequence of Lemmas 3.1 and 3.2. By (9.7), $\lim _{i, j \rightarrow \infty} \bar{g}_{i, j}$ $=\lim _{i, j \rightarrow \infty} \bar{h}_{i, j}=0$, with respect to convergence in measure and hence with respect to $L^{q}$ norm for every $q \in[1, \infty)$. Also, in case $(\mathrm{a}),\left\{g_{i, j}-\bar{g}_{i, j}\right\}_{i, j \geqslant n_{0}}$ and $\left\{h_{i, j}-\bar{h}_{i, j}\right\}_{i, j \geqslant n_{0}}$ are bounded families of functions. Hence, by $(9.5),(9.7)$ and the continuity property of $N$, we obtain

$$
\lim _{i, j \rightarrow \infty} N\left(g_{i, j}-\bar{g}_{i, j}\right)=\lim _{i, j \rightarrow \infty} N\left(h_{i, j}-\bar{h}_{i, j}\right)=N\left(f^{*}\right) .
$$

Note that, by (9.2), (9.4) and (9.7)

$$
K\left(g_{i, j}\right) \cap K\left(\bar{g}_{i, j}\right)=K\left(h_{i, j}\right) \cap K\left(\bar{h}_{i, j}\right)=\varnothing .
$$

Hence, by (9.3) and (4.2)

$$
\begin{aligned}
I_{i, j} & =\int_{I}\left[\mathbf{H}\left(g_{i, j}-\bar{g}_{i, j}\right)-\mathbf{H}\left(h_{i, j}-\bar{h}_{i, j}\right)\right] d m-I_{i, j}^{\prime} \\
& =N\left(g_{i, j}-\bar{g}_{i, j}\right)-N\left(h_{i, j}-\bar{h}_{i, j}\right)-I_{i, j}^{\prime}
\end{aligned}
$$


where $I_{i, j}^{\prime}=\int_{I}\left[\mathbf{H}\left(-\bar{g}_{i, j}\right)-\mathbf{H}\left(-\bar{h}_{i, j}\right)\right] d m$. In view of the fact that $H(\cdot, 1)$ and $H(\cdot,-1)$ belong to $L^{1}(I),(9.7)$ implies that $\lim _{i, j \rightarrow \infty} I_{i . j}^{\prime}=0$. Hence, by (9.8) and (9.9), $\lim _{i, j \rightarrow \infty} I_{i, j}=0$. This completes the proof of the lemma.

We are ready now to complete the proof of Theorem 1.3.

Proof of Theorem 1.3. Let $H$ be a kernel for $N$ in the sense of Theorem 4.1. We define an operator $\mathcal{H}: L^{p}(0,1) \rightarrow L^{1}(0,1)$ as follows.

Given $f$ in $L^{p}(0,1)$, select a sequence of simple functions $\left\{f_{n}\right\}$ which converges to $f$ with respect to the $L^{p}$ norm. Set

$$
\mathfrak{H}(f)=L^{1}-\lim _{n \rightarrow \infty} \mathbf{H}\left(f_{n}\right) .
$$

By Lemma 9.1 the limit exists and is independent of the choice of the approximating sequence $\left\{f_{n}\right\}$. Moreover

$$
\mathcal{K}(f)=\mathbf{H}(f) \text { whenever } f \text { is a simple measurable function. }
$$

The operator $\mathcal{K}$ possesses the following properties:

$(\alpha) \mathcal{H}\left(f \chi_{E}\right)=\mathscr{H}(f) \chi_{E}, \forall f \in L^{p}(I), \forall E \in \tau$;

$(\beta) \mathfrak{H}$ is continuous with respect to the $L^{p}$ norm if $1 \leqslant p<\infty$ and with respect to the $(b m)$-topology if $p=\infty$.

This follows from the fact that $H(\cdot, 0)=0$ and from Lemma 9.1(a), (b).

By [5, Theorem 3.3], properties $(\alpha)$ and $(\beta)$ imply the existence of a Carathéodory function $H^{*}:[0,1] \times R \rightarrow R$ such that

$$
\mathfrak{H}(f)=\mathbf{H}^{*}(f), \quad \forall f \in L^{p}(0,1) .
$$

By (9.11) and (9.12), $\mathbf{H}(f)=\mathbf{H}^{*}(f)$ whenever $f$ is a simple measurable function. Therefore $H^{*}$ is also a kernel for $N$ (in the sense of Theorem 4.1). Thus we have

$$
N(f)=\int_{I} \mathbf{H}^{*}(f) d m, \quad \forall f \in \varsigma_{k} .
$$

Now let $f \in \mathfrak{N R}_{k}^{p}$. By Lemma A.2 (see Appendix) there exists a sequence $\left\{f_{n}\right\}$ in $s_{k}$ which converges to $f$ in $L^{p}(I)$. By $(\beta) \mathcal{H}\left(f_{n}\right) \rightarrow \mathcal{H}(f)$ in $L^{1}(I)$. By property (ii) of $N, N\left(f_{n}\right) \rightarrow N(f)$. Hence (9.12) and (9.13) imply that

$$
N(f)=\int_{l} \mathbf{H}^{*}(f) d m, \quad \forall f \in \mathbb{O}_{k}^{p} .
$$

This completes the proof of the existence of a Carathéodory kernel for $N$.

The characterization of the set of all Carathéodory kernels for $N$, as stated in the theorem, is an immediate consequence of the parallel characterization in Theorem 4.1. Finally, the fact that conditions (i) and (ii) are necessary for the existence of a Carathéodory kernel, has already been proved in $\$ 1$.

This completes the proof of the theorem. 
10. The representation theorem for operators. In this section we prove Theorem 1.4. But, first we give two auxiliary results.

LEMMA 10.1. Let $\mathcal{H C}_{:} \mathfrak{S}_{k} \rightarrow L^{1}(I)$ be an odd disjointly additive operator súch that

$$
K(\mathcal{H}(f)) \subseteq K(f), \quad \forall f \in \mathcal{S}_{k} .
$$

Let $h, f_{1}, f_{2}$ be simple functions such that

$$
\left\{\begin{array}{l}
\text { (a) } K(h) \cap\left(K\left(f_{1}\right) \cup K\left(f_{2}\right)\right)=\varnothing, \\
\text { (b) } \hat{\mu}_{k}(h)=\hat{\mu}_{k}\left(f_{1}\right)=\hat{\mu}_{k}\left(f_{2}\right) .
\end{array}\right.
$$

Set $U=K(h)$. Then

$$
\mathcal{H}\left(f_{1}-h\right) \chi_{U}=\mathcal{H}\left(f_{2}-h\right) \chi_{U}
$$

Proof. First assume that in addition to (10.2), the functions $f_{1}, f_{2}$ satisfy the condition

$$
K\left(f_{1}\right) \cap K\left(f_{2}\right)=\varnothing
$$

Then, by the same argument that was used in the proof of Lemma 2.2, we have

$$
\mathcal{H}\left(f_{1}-f_{2}\right)=\mathcal{H}\left(f_{1}-h\right)-\mathcal{H}\left(f_{2}-h\right)
$$

Clearly, (10.1) and (10.5) imply (10.3).

Now, consider the general case where $f_{1}, f_{2}$ are any two simple functions satisfying (10.2).

Let

$$
f_{i}=\sum_{j=1}^{\nu_{i}} a_{i, j} \chi_{S_{i, j}}, \quad(i=1,2)
$$

where $\left\{S_{i, j}\right\}_{j=1}^{\nu_{i}}$ is a partition of $I$. Set $W_{l, n}=S_{1, l} \cap S_{2, n}, 1 \leqslant l \leqslant \nu_{1}, 1 \leqslant n$ $\leqslant \nu_{2}$. Let $\left\{W_{l, n}^{\prime}, W_{l, n}^{\prime \prime}\right\}$ be a partition of $W_{l, n}$ into sets of equal $\mu_{k}$ measure and define

$$
\left\{\begin{aligned}
f_{i}^{\prime}=\sum_{l, n} b_{i, l, n} \chi_{W_{l, n}^{\prime}}, \quad f_{i}^{\prime \prime}=\sum b_{i, l, n} \chi_{W_{l, n}^{\prime \prime}} \quad(i=1,2), \\
\text { where } b_{1, l, n}=a_{1, l}, b_{2, l, n}=a_{2, n} .
\end{aligned}\right.
$$

Then we have

$$
\begin{cases}f_{i}=f_{i}^{\prime}+f_{i}^{\prime \prime}, \quad \hat{\mu}_{k}\left(f_{i}^{\prime}\right)=\hat{\mu}_{k}\left(f_{i}^{\prime \prime}\right), & (i=1,2) \\ K\left(f_{i}^{\prime}\right) \cap K\left(f_{j}^{\prime \prime}\right)=\varnothing, & (i, j=1,2)\end{cases}
$$


We consider also an arbitrary $\mu_{k}$-uniform decomposition of $h$, say $\left\{h^{\prime}, h^{\prime \prime}\right\}$ and denote $U^{\prime}=K\left(h^{\prime}\right), U^{\prime \prime}=K\left(h^{\prime \prime}\right)$. Then, by $(10.6)$ and the result proved in the first part of the proof, we have

$$
\mathcal{H}\left(f_{1}^{\prime}-h^{\prime}\right) \chi_{U^{\prime}}=\mathcal{H}\left(f_{2}^{\prime \prime}-h^{\prime}\right) \chi_{U^{\prime}}, \quad \mathcal{H}\left(f_{1}^{\prime \prime}-h^{\prime \prime}\right) \chi_{U^{\prime \prime}}=\mathcal{H}\left(f_{2}^{\prime}-h^{\prime \prime}\right) \chi_{U^{\prime \prime}} .
$$

In view of (10.1), these inequalities remain valid if we replace $\chi_{U^{\prime}}$ and $\chi_{U^{\prime \prime}}$ by $\chi_{U},(U=K(h))$. Hence, summing up the two equalities, we get (10.3).

LEMMA 10.2. Let $\mathcal{H}_{:} \delta_{k} \rightarrow L^{1}(I)$ be a disjointly additive operator satisfying condition (10.1).

Then, there exists a function $H: I \times R \rightarrow R$ such that

$$
\left\{\begin{array}{l}
H(\cdot, a) \in L^{l}(I), \quad \forall a \in R, \\
H(\cdot, 0)=0
\end{array}\right.
$$

and

$$
\mathcal{H}(f)=\mathbf{H}(f), \quad \forall f \in \delta_{k} .
$$

Proof. The operator $\mathcal{H}$ may be written as the sum of an odd operator and an even operator, each satisfying the assumptions of the lemma. Therefore it is sufficient to prove this lemma in the case where $\mathcal{H}$ is odd and in the case where $\mathcal{H}$ is even.

Suppose that $\mathcal{H}$ is odd. Let $a \in R$ and let $U$ be a set in $\tau$ with $m(U)<1$. Then there exists a simple function $g$ such that

$$
K(g) \cap U=\varnothing \text { and } \hat{\mu}_{k}(g)=a \mu_{k}(U) .
$$

This is a consequence of Lemmas 3.1 and 3.2.

By Lemma 10.1 the element of $L^{1}(I)$ given by $\mathscr{C}\left(a \chi_{U}-g\right) \chi_{U}$ is uniquely determined by $U$ (provided that $g$ satisfies (10.9)). Thus for any $a$ and $U$ as above we set

$$
H_{U}(\cdot, a)=\mathcal{H}\left(a \chi_{U}-g\right) \chi_{U}
$$

where $g$ is any simple function satisfying (10.9). If $V$ is a measurable subset of $U$ then

$$
H_{U}(\cdot, a) \chi_{V}=\mathcal{H}\left(a \chi_{U}-g\right) \chi_{V}=\mathcal{H}\left(a \chi_{V}-\left(g-a \chi_{U \backslash V}\right)\right) \chi_{V}
$$

so that

$$
H_{V}(\cdot, a)=H_{U}(\cdot, a) \chi_{V}
$$


Now let $\left\{U_{1}, U_{2}\right\}$ be a partition of $I$ into measurable sets of positive measure. Then $H_{l_{1}}(\cdot, a)+H_{U_{2}}(\cdot, a)$ as an element of $L^{1}(I)$ is independent of the particular choice of the partition. This is a simple consequence of (10.11). Set

$$
H(\cdot, a)=H_{l_{1}}(\cdot, a)+H_{l_{2}}(\cdot, a), \quad \forall a \in R
$$

From (10.11) and (10.12) we obtain

$$
H_{l^{\prime}}(\cdot, a)=H(\cdot, a) \chi_{l}, \quad \forall U \in \tau, \forall a \in R .
$$

Let $H: I \times R \rightarrow R$ be the function defined by (10.12). Then clearly it satisfies (10.7). Also, (10.10) and (10.13) imply (10.8).

Now consider the case where $\mathcal{H}$ is even. If $U \in \tau$ and $\left\{U_{1}, U_{2}\right\}$ is a partition of $U$ into sets of equal $\mu_{k}$ measure, then $\mathcal{H}\left(a\left(\chi_{l_{1}}-\chi_{L_{2}^{\prime}}\right)\right),(a \in R)$, is independent of the particular choice of the partition. This is proved in the same way as formula (2.5) (see proof of Lemma 2.1). Set

$$
H_{l^{\prime}}(\cdot, a)=\mathscr{H}\left(a\left(\chi_{l_{1}}-\chi_{l_{2}}\right)\right), \quad \forall a \in R
$$

If we denote $H_{l}(\cdot, a)$ by $H(\cdot, a)$, then

$$
H_{L^{\prime}}(\cdot, a)=H(\cdot, a) \chi_{L^{\prime}}, \quad \forall U \in \tau, \forall a \in R .
$$

Indeed, by (10.1) and (10.14), $K\left(H_{L^{\prime}}(\cdot, a)\right) \subseteq U$. Also if $U^{\prime} \in \tau$ and $V=I \backslash U$, then (by (10.14) and disjoint additivity), $H_{l} \cdot(\cdot, a)+H_{l}(\cdot, a)=H(\cdot, a)$. These two facts imply (10.15).

Clearly, the function $H: I \times R \rightarrow R$ defined above satisfies condition (10.7). (Note that $(10.1)$ implies that $\mathscr{H}(0)=0$, so that $H(\cdot, 0)=0$.) We proceed to show that it also satisfies condition (10.8).

Let $f \in \varsigma_{k}, f=\sum_{i=1}^{n} a_{i} \chi_{S_{i}}$, where $\left\{S_{i}\right\}_{1}^{n}$ is a partition of $I$. Let $\left\{S_{i}^{\prime}, S_{i}^{\prime \prime}\right\}$ be a partition of $S_{i}$ into sets of equal $\mu_{k}$ measure and set

$$
f^{\prime}=\sum_{i=1}^{n} a_{i} \chi_{S_{i}^{\prime}}, \quad f^{\prime \prime}=\sum_{i=1}^{n} a_{i} \chi_{S_{i}^{\prime \prime}}
$$

In view of the fact the $\mathcal{H}$ is disjointly additive and even, we have

$$
\begin{aligned}
\mathscr{H}(f) & =\mathscr{H}\left(f^{\prime}\right)+\mathscr{H}\left(f^{\prime \prime}\right)=\mathscr{H}\left(f^{\prime}-f^{\prime \prime}\right) \\
& =\sum_{1}^{n} \mathscr{H}\left(a_{i}\left(\chi_{S_{i}^{\prime}}-\chi_{S^{\prime \prime}}\right)\right) \\
& =\sum_{1}^{n} H_{S_{i}}\left(\cdot, a_{i}\right)=\mathbf{H}(f) .
\end{aligned}
$$

This completes the proof of the lemma.

We turn now to 
Proof of Theorem 1.4. Let $H$ be a function satisfying (10.7) and (10.8) with respect to the given operator $\mathcal{H}$. If $f$ is a measurable simple function, then $\mathbf{H}(f) \in L^{l}(I)$.

We claim that $H$ possesses the following properties:

(a) If $p=\infty$ and if $\left\{f_{n}\right\}_{1}^{\infty}$ is a $(\mathrm{bm})$-convergent sequence of simple functions, then $\left\{\mathbf{H}\left(f_{n}\right)\right\}_{1}^{\infty}$ is a Cauchy sequence in $L^{1}(I)$.

(b) If $1 \leqslant p<\infty$ and if $\left\{f_{n}\right\}_{1}^{\infty}$ is an $L^{p}$-convergent sequence of simple functions, then $\left\{\mathbf{H}\left(f_{n}\right)\right\}_{1}^{\infty}$ is a Cauchy sequence in $L^{1}(I)$.

(c) In both cases, if the limit of $\left\{f_{n}\right\}$ is a simple function $f$, then $\mathbf{H}\left(f_{n}\right)$ $\rightarrow \mathbf{H}(f)$ in $L^{1}(I)$.

Let $D=\cup_{1}^{\infty} K\left(f_{n}\right)$. Clearly it is sufficient to prove these assertions in the case where $m(D) \leqslant \frac{1}{2}$. Let $f=\lim f_{n}$, with respect to $(\mathrm{bm})$-convergence in (a) and $L^{p}$-convergence in (b). If $M$ is a sufficiently large positive number, then there exists a function $h$ such that

$$
\left\{\begin{array}{l}
h=M\left(\chi_{U}-\chi_{V}\right), \quad m(K(h)) \leqslant \frac{1}{4}, \quad K(h) \cap D=\varnothing \\
\hat{\mu}_{k}(h)=\hat{\mu}_{k}(f) .
\end{array}\right.
$$

This is a consequence of Lemmas 3.1 and 3.2.

Set

$$
f^{*}=f-h, \quad f_{n}^{*}=f_{n}-h .
$$

Note that $\lim f_{n}^{*}=f^{*}$, with respect to $(b m)$-convergence in (a) and $L^{p}$ convergence in (b). Hence, in both cases,

$$
\hat{\mu}_{k}\left(f_{n}^{*}\right) \rightarrow \hat{\mu}_{k}\left(f^{*}\right)=0 .
$$

Let $D^{*}=\cup_{1}^{\infty} K\left(f_{n}^{*}\right)$; note that $m\left(D^{*}\right) \leqslant \frac{3}{4}$. In view of $(10.18)$, if $n$ is sufficiently large, (say $n \geqslant n_{0}$ ), then there exists a function $h_{n}$ such that

$$
\left\{\begin{array}{l}
h_{n}=\chi_{U_{n}}-\chi_{V_{n}}, \quad K\left(h_{n}\right) \cap D^{*}=\varnothing, \quad \hat{\mu}_{k}\left(h_{n}\right)=\hat{\mu}_{k}\left(f_{n}^{*}\right), \\
m\left(K\left(h_{n}\right)\right) \leqslant c_{0}\left|\hat{\mu}_{k}\left(f_{n}^{*}\right)\right|^{\prime},
\end{array}\right.
$$

where $c_{0}$ is a constant independent of $n$. (We recall that for $x=\left(x_{1}, \ldots, x_{k}\right)$ $\in R_{k},|x|^{\prime}=\max \left(\left|x_{1}\right|, \ldots,\left|x_{k}\right|\right)$.) Again, this is a consequence of Lemmas 3.1 and 3.2. Further, (10.17)-(10.19) imply that

$$
\lim h_{n}=0 \text { and } \lim \left(f_{n}^{*}-h_{n}\right)=f^{*},
$$

with respect to $(\mathrm{bm})$-convergence in (a) and $L^{p}$-convergence in (b). Moreover $\left(f_{n}^{*}-h_{n}\right) \in \varsigma_{k},\left(n \geqslant n_{0}\right)$. Hence, by assumption (ii),

$$
\mathcal{K}\left(f_{n}^{*}-h_{n}\right) \rightarrow \mathcal{H}\left(f^{*}\right) \text { in } L^{\prime}(I) .
$$


Therefore, by (10.8), $\left\{\mathbf{H}\left(f_{n}^{*}-h_{n}\right)\right\}_{n_{0}}^{\infty}$ is a Cauchy sequence in $L^{1}(I)$. However, by (10.7), $\mathbf{H}\left(f_{n}\right)=\left(\mathbf{H}\left(f_{n}^{*}-h_{n}\right)\right) \chi_{D},\left(n \geqslant n_{0}\right)$. Thus $\left\{\mathbf{H}\left(f_{n}\right)\right\}$ is a Cauchy sequence in $L^{1}(I)$. This proves assertions (a) and (b). Once these assertions are proved, (c) is obvious.

Now let $f \in L^{P}(I)$ and let $\left\{f_{n}\right\}$ be a sequence of measurable simple functions converging to $f$ in $L^{p}(I)$. Then, by (a) and (b), the $L^{1}$ limit of $\left\{\mathbf{H}\left(f_{n}\right)\right\}$ exists and is independent of the particular choice of the approximating sequence $\left\{f_{n}\right\}$. Set

$$
\mathcal{H}^{*}(f)=L^{1}-\lim \mathbf{H}\left(f_{n}\right)
$$

The operator $\mathcal{H}^{*}: L^{p}(I) \rightarrow L^{1}(I)$, defined by the above formula, possesses the following two properties:

$(\alpha) \mathcal{H}^{*}\left(f \chi_{E}\right)=\mathcal{H}^{*}(f) \chi_{E}, \quad \forall f \in L^{p}(I), \forall E \in \tau$;

(B) $\mathcal{T}^{*}$ is continuous with respect to the $L^{p}$ norm if $1 \leqslant p<\infty$ and with respect to the $(b m)$ topology if $p=\infty$.

This follows from the fact that $H(\cdot, 0)=0$ and from properties (a) and (b) of H.

By [5, Theorem 3.3], $(\alpha)$ and $(\beta)$ imply that there exists a Carathéodory function $H^{*}: I \times R \rightarrow R$ such that

$$
\mathcal{H}^{*}(f)=\mathbf{H}^{*}(f), \quad \forall f \in L^{p}(I) .
$$

By property (c) and (10.21),

$$
\mathcal{K}^{*}(f)=\mathbf{H}(f) \text { whenever } f \text { is a simple function in } L^{p}(I) .
$$

In particular we deduce that, $H(\cdot, a)=H^{*}(\cdot, a)$ for every real $a$, the equality being understood as Lebesgue equivalence. By (10.8) and (10.23)

$$
\mathcal{H}^{*}(f)=\mathcal{H}(f), \quad \forall f \in \mathcal{S}_{k} .
$$

Since $\delta_{k}$ is dense in $\Re_{k}^{p}$ with respect to the $L^{p}$ norm $(1 \leqslant p \leqslant \infty)$ (see Appendix), (10.24) and the continuity properties of $\mathcal{K}$ and $\mathcal{H}^{*}$ imply that $\mathcal{H}^{*}(f)=\mathcal{H}(f), \forall f \in \mathscr{T}_{k}^{p}$. Finally, by $(10.22)$,

$$
\mathcal{H}(f)=\mathbf{H}^{*}(f), \quad \forall j \in \mathscr{T}_{k}^{p} .
$$

Thus $H^{*}$ is a normalized function in $\mathrm{Car}^{p}$ satisfying (1.7). It is easily seen that these properties determine $H^{*}$ uniquely up to equivalence.

The fact that the assumptions (i)-(iii) are necessary was shown in $\S 1$. This completes the proof of the theorem.

Appendix. The object of this appendix is to provide proofs of two auxiliary results that were used in previous sections. First we shall show that $\mathrm{sp} \mathcal{E}_{k}^{0}$ is 
dense in $\Re_{k}^{p}$. Then, using this result, we shall prove Lemma 6.5 .

LEMma A.1. Let $f$ be a rational valued function in $\mathcal{S}_{k}$. Then $f \in \operatorname{sp} \varepsilon_{k}^{0}$.

Proof. First we consider the case $k=1$. It can be verified that every function $f$ in $\delta_{1}$ can be written as a sum, $f=h_{1}+\cdots+h_{n}$, such that $h_{1}, \ldots, h_{n}$ are disjoint functions in $\mathscr{E}_{1}$. If, in addition, $f$ is rational valued then $h_{1}, \ldots, h_{n}$ will be rational valued. But, it is easily seen that every rational valued function in $\mathcal{E}_{1}$ belongs to sp $\mathcal{E}_{1}^{0}$. Therefore the lemma holds in the case $k=1$. Now, consider the case $k>1$. Without loss of generality, we may assume that

$$
m(K(f)) \leqslant \rho_{k}\left(\frac{1}{2}\right),
$$

with $\rho_{k}(\alpha)$ as in Lemma 3.1. Otherwise we consider a $\mu_{k}$-uniform decomposition of $f$.

Thus, we assume that $f$ satisfies (A.1). Let $D=I \backslash K(f)$. Since $\rho_{k}\left(\frac{1}{2}\right)$ $\leqslant \frac{1}{2}$ we have $m(D) \geqslant \frac{1}{2}$. By Lemma 3.1 , there exists a $\mu_{k}$-independent family of sets $\mathbb{Q}=\left\{A_{1}, \ldots, A_{k}\right\}$ such that $A_{1}, \ldots, A_{k} \subset D$ and $\rho(\mathbb{Q}) \geqslant \rho_{k}\left(\frac{1}{2}\right)$. Let $\left\{\Sigma_{j}\right\}_{1}^{k}$ and $\Sigma$ be as in Definition 3.2. By Corollary 3.3, there exists a $\Sigma$ representative of $f$, which we shall denote by $\tilde{f}$. Clearly $f-\tilde{f} \in \operatorname{sp} \mathscr{E}_{k}^{0}$. Therefore, the proof will be completed if we show that $\tilde{f} \in \mathrm{sp} \varepsilon_{k}^{0}$.

Set $\tilde{f}=\Sigma_{1}^{k} g_{j}$ where $g_{j}$ is a $\Sigma_{j}$-measurable simple function $(j=1, \ldots, k)$. Then $g_{j}$ is rational valued and, by Lemma $3.4, g_{j} \in \delta_{k}$. Now, on $\Sigma_{j}$ we have,

$$
\mu_{k}(U)=m(U)\left[\mu_{k}\left(A_{j}\right) / m\left(A_{j}\right)\right] .
$$

Moreover, the measure space $\left(A_{j}, \Sigma_{j}, m\right)$ is isomorphic (modulo will sets) to the Lebesgue measure space in $\left[0, m\left(A_{j}\right)\right],[6, \mathrm{p} .173]$. Therefore, the validity of the lemma in the case $k=1$ implies that every rational valued function in $\delta_{k}\left(\Sigma_{j}\right)$ belongs to $\operatorname{sp} \varepsilon_{k}^{0}\left(\Sigma_{j}\right)$. In particular $g_{j}$ belongs to $\operatorname{sp} \varepsilon_{k}^{0}\left(\Sigma_{j}\right),(j=1, \ldots, k)$. This shows that $\tilde{f} \in \mathrm{sp} \mathscr{E}_{k}^{0}$ and the proof is complete.

Lemma A.2. The linear manifold $\mathrm{sp} \varepsilon_{k}^{0}$ is dense in $\mathfrak{\pi}_{k}^{p}$ with respect to the $L^{p}$ norm, $1 \leqslant p \leqslant \infty$.

Proof. In view of Lemma A.1 it is sufficient to show that $\delta_{k}^{\text {rat }}=\left\{f \in \delta_{k}: f\right.$ is rational valued $\}$ is dense in $\pi_{k}^{p}$.

Let $f \in \pi_{k}^{p}$ and let $\left\{f_{n}\right\}$ be a sequence of rational valued simple functions which converge to $f$ in $L^{p}(I)$. If there exists a subsequence $\left\{f_{n_{i}}\right\}$ such that $\hat{\mu}_{k}\left(f_{n_{i}}\right)=0$ there is nothing more to prove. Therefore we may assume that $\hat{\mu}_{k}\left(f_{n}\right) \neq 0$ for all $n$.

Let $Q$ be a $\mu_{k}$-independent family of sets and let $\Sigma$ be as in Definition 3.2. Let $r_{n}$ be a rational number such that 


$$
\left|\hat{\mu}_{k}\left(f_{n}\right)\right|^{\prime} / \rho(\hat{A}) \leqslant r_{n} \leqslant 2\left|\hat{\mu}_{k}\left(f_{n}\right)\right|^{\prime} / \rho\left(\hat{A}^{\prime}\right) .
$$

Set $y^{(n)}=r_{n}^{-1} \hat{\mu}_{k}\left(f_{n}\right)$. Then $\left|y^{(n)}\right|^{\prime} \leqslant \rho\left(\hat{\psi}^{\prime}\right)$ and, by Lemma 3.2, there exists a $\Sigma$-representative for $y^{(n)}$, say $h_{n}^{\prime}$. Let $h_{n}=r_{n} h_{n}^{\prime}$. Then $\max _{I}\left|h_{n}\right|=r_{n} \rightarrow 0$. The convergence to zero follows from the fact that $\hat{\mu}_{k}\left(f_{n}\right) \rightarrow \hat{\mu}_{k}(f)=0$. Thus, $f_{n}-h_{n} \in \mathbb{s}_{k}^{\text {rat }}$ and $f_{n}-h_{n} \rightarrow f$ in $L^{p}(I)$. This completes the proof of the lemma.

Finally we provide

Proof of Lemma 6.5. The assumption (6.20) and Lemma A.2 imply that

$$
\int_{I} g f d m=0, \quad \forall f \in \Omega_{k}^{\infty}
$$

Suppose that $g \notin P_{k}=$ space of polynomials of order less than $k$. Then, by the Hahn-Banach theorem, there exists a continuous linear functional on $L^{\prime}(I)$ which vanishes on $P_{k}$ but does not vanish at $g$. Thus, there exists an element $f_{0}$ in $L^{\infty}(I)$ such that

$$
\int_{I} f_{0} g d m \neq 0 \text { and } \int_{I} f_{0} q d m=0, \quad \forall q \in P_{k}
$$

The second of these relations means that $f_{0} \in \mathcal{O}_{k}^{\infty}$. Therefore (A.3) contradicts (A.2).

\section{REFERENCES}

1. R. A. Alo and A. de Korvin, Representation of Hammerstein operators by Nemytskii measures, J. Math. Anal. Appl. 52 (1975), 490-513.

2. J. Batt, Nonlinear integral operators on $C(S, E)$, Studia Math. 48 (1973), 145-177. MR 49 \#1110.

3. D. H. Blackwell, The range of certain vector integrals, Proc. Amer. Math. Soc. 2 (1951), 390-395. MR 12, 810.

4. R. V. Chacon and N. Friedman, Additive functionals, Arch. Rational Mech. Anal. 18 (1965), 230-240. MR 30 \#2329.

5. L. Drewnowski and $\mathrm{W}$. Orlicz, Continuity and representation of orthogonally additive functionals, Bull. Acad. Polon. Sci. Sér. Sci. Math. Astronom. Phys. 17 (1969), 647-653. MR 41 \#806.

6. P. R. Halmos, Measure theory, Van Nostrand, Princeton, N. J. 1950. MR 11, 504.

7. M. A. Krasnosel'skiī, Topological methods in the theory of nonlinear integral equations, GITTL, Moscow, 1956; English transl., Macmillan, New York, 1964. MR 20 \#3464; 28 \#2414.

8. M. A. Krasnosel'skiī, D. P. Zabreiko, E. I. Pustyl'nik and P. E. Sobolevskiī, Integral operators in spaces of summable functions, "Nauka", Moscow, 1966; English transl., Noordhoff, Leyden, 1976. MR 34 \#6568.

9. A. Lyapunov, Sur les fonctions-vecteurs complètement additives, Izv. Akad. Nauk SSSR Ser. Mat. 4 (1940), 465-478. MR 21, 315. 
10. A. D. Martin and V. J. Mizel, A representation theorem for certain nonlinear functionals, Arch. Rational Mech. Anal. 15 (1964), 353-367. MR 28 \#3330.

11. V. J. Mizel, Characterization of non-linear transformations possessing kernels, Canad. J. Math. 22 (1970), 449-471. MR 41 \#495.

12. V. J. Mizel and K. Sundaresan, Representation of vector valued nonlinear functions, Trans. Amer. Math. Soc. 159 (1971), 111-127. MR 43 \#5368.

Department of Mathematics, Carnegie-Mellon University, Pittsburgh, Pennsylvania 15213

Current address (both authors): Department of Mathematics, Technion-Israel Institute of Technology, Haifa, Israel 